Illinois State University

ISU ReD: Research and eData

Theses and Dissertations

3-12-2021

\title{
Modeling Individual Muscle Forces In The Lower Extremity During Loaded And Unloaded Hexbar Vertical Jumps
}

Abigail Salvadore

Illinois State University, bballkid1126@gmail.com

Follow this and additional works at: https://ir.library.illinoisstate.edu/etd

Part of the Biomechanics Commons, and the Physiology Commons

\section{Recommended Citation}

Salvadore, Abigail, "Modeling Individual Muscle Forces In The Lower Extremity During Loaded And Unloaded Hexbar Vertical Jumps" (2021). Theses and Dissertations. 1408.

https://ir.library.illinoisstate.edu/etd/1408

This Thesis is brought to you for free and open access by ISU ReD: Research and eData. It has been accepted for inclusion in Theses and Dissertations by an authorized administrator of ISU ReD: Research and eData. For more information, please contact ISUReD@ilstu.edu. 


\section{MODELING INDIVIDUAL MUSCLE FORCES IN THE LOWER EXTREMITY DURING LOADED AND UNLOADED HEXBAR VERTICAL JUMPS}

\section{ABIGAIL K. SALVADORE}

58 Pages

Hexagonal barbell (HB) loaded jumps are often used in training to increase lower extremity power. Given the importance of coordinated muscular effort in achieving maximal power output, an understanding of how the lower extremity musculature individually performs during loaded jumps would be advantageous. The purpose of this study is to describe the effect of load on individual muscle forces, muscle torques, and the contribution to the net joint moment (NJM) during the concentric phase of loaded HB jumps.

10 male collegiate athletes performed 5 maximal HB jumps at $0 \%, 20 \%, 40 \%$ and $60 \%$ of their HB deadlift 1-repetition maximum. Filtered Ground reaction forces and 3D lower extremity marker trajectories were input into a 23 DOF musculoskeletal model and muscle forces were estimated with static optimization. Peak muscle force $(\mathrm{xBW})$ was calculated for the gluteus maximum (GMAX), biceps femoris - long head (BFL), rectus femoris (RF), vastus intermedius (VAST), gastrocnemius (GAS), and soleus (SOL). RMANOVA and LSD comparisons were used for analysis $(\mathrm{p}<0.05)$. Muscle torque $(\mathrm{Nm} / \mathrm{kg})$ and the contribution to the NJM was calculated for each muscle and analyzed qualitatively.

A significant increase in peak muscle force across loads existed for VAST $(p=0.009)$ and GAS $(p<0.001)$, and significant decreases were noted for RF $(p=0.017)$. There was no significant difference in peak force of GMAX $(p=0.325)$, BFL $(p=0.369)$, or SOL $(p=0.122)$ 
across loads. Torque contribution from individual muscles was unaltered at the ankle but shifted towards the vasti at the knee and the extensors at the hip with increasing loads. Loaded hexbar jumping is not simply a higher intensity version of vertical jumping, and the lower extremity joints and corresponding musculature are not impacted equally by the addition of load. The varied effect of load on mechanical demands at the lower extremity joints, and thus force and torque output from individual muscles, is important to consider when using loaded jumps as part of training for athletic performance.

KEYWORDS: Musculoskeletal Modeling, Muscle Force, Jumping, Hexbar 
MODELING INDIVIDUAL MUSCLE FORCES IN THE LOWER EXTREMITY

DURING LOADED AND UNLOADED HEXBAR VERTICAL JUMPS

ABIGAIL K. SALVADORE

A Thesis Submitted in Partial

Fulfillment of the Requirements for the Degree of

MASTER OF SCIENCE

School of Kinesiology and Recreation

ILLINOIS STATE UNIVERSITY 
Copyright 2021 Abigail K. Salvadore 


\title{
MODELING INDIVIDUAL MUSCLE FORCES IN THE LOWER EXTREMITY DURING LOADED AND UNLOADED HEXBAR VERTICAL JUMPS
}

\author{
ABIGAIL K. SALVADORE
}

COMMITTEE MEMBERS:

Michael Torry, Chair

Adam Jagodinsky 


\section{ACKNOWLEDGMENTS}

I want to thank Dr. Torry and Dr. J. for their guidance and mentorship over the last 2 years and on this thesis. You gave me plenty of room to run and figure things out on my own but were also available to help or redirect as needed. I owe a huge thank you to Sean Higinbotham for all his help with OpenSim over the summer, and I would also like to thank Kyle Pigney and Drew Himmelman for their help with preparing the data for OpenSim and comparing my outputs to Visual3D. Additionally, I would like to thank Dr. Kristof Kipp for his correspondence regarding the contribution data. Lastly, I want to acknowledge my family, friends, and everyone else in my circle. You guys kept me sane and on-track throughout my time at ISU, and I couldn't have done this without you in my corner.

A. K. S. 


\section{CONTENTS}

Page

ACKNOWLEDGMENTS

TABLES

FIGURES

CHAPTER I: MODELING INDIVIDUAL MUSCLE FORCES DURING LOADED AND

UNLOADED HEXBAR VERTICAL JUMPS 1

$\begin{array}{ll}\text { Introduction } & 1\end{array}$

$\begin{array}{ll}\text { Methods } & 4\end{array}$

$\begin{array}{ll}\text { Subjects } & 4\end{array}$

$\begin{array}{ll}\text { Instrumentation } & 4\end{array}$

$\begin{array}{ll}\text { Procedures } & 5\end{array}$

Data Collection $\quad 5$

$\begin{array}{ll}\text { Data Processing } & 6\end{array}$

$\begin{array}{ll}\text { Musculoskeletal Modeling } & 6\end{array}$

$\begin{array}{ll}\text { Scaling } & 7\end{array}$

$\begin{array}{ll}\text { Dynamic Analyses } & 7\end{array}$

$\begin{array}{ll}\text { Data Analysis } & 8\end{array}$

Statistical Analysis $\quad 9$

$\begin{array}{ll}\text { Results } & 10\end{array}$

$\begin{array}{ll}\text { Individual Muscle Forces } & 14\end{array}$

$\begin{array}{ll}\text { Individual Muscle Torques and Contributions } & 19\end{array}$

$\begin{array}{ll}\text { Hip } & 20\end{array}$ 
$\begin{array}{ll}\text { Ankle } & 28\end{array}$

$\begin{array}{ll}\text { Discussion } & 31\end{array}$

$\begin{array}{ll}\text { Muscle Forces } & 34\end{array}$

$\begin{array}{ll}\text { Muscle Torques } & 37\end{array}$

$\begin{array}{ll}\text { Limitations } & 43\end{array}$

$\begin{array}{ll}\text { Conclusion } & 45\end{array}$

REFERENCES 46

APPENDIX A: MARKER PLACEMENT AND LOCATIONS 54

APPENDIX B: DETAILED WORKFLOW 55 


\section{TABLES}

Table

Page

1. Relative Peak Muscle Forces Across Loads

2. Absolute Peak Muscle Forces Across Loads

3. Peak Muscle Forces as Compared to Literature 


\section{FIGURES}

Figure $\quad$ Page

1. Peak Height Above the Ground of the Sacrum Marker Across Loading Conditions $\quad 10$

2. Hip Joint Angle During the Concentric Phase 11

3. Knee Joint Angle During the Concentric Phase 11

4. Ankle Joint Angle During the Concentric Phase 12

5. Relative Hip Net Joint Moment During the Concentric Phase 13

6. Relative Knee Net Joint Moment During the Concentric Phase 13

7. Relative Ankle Net Joint Moment During the Concentric Phase 14

8. Relative Gluteus Maximus Force During the Concentric Phase 16

9. Relative Biceps Femoris - Long Head During the Concentric Phase 16

10. Relative Soleus Force During the Concentric Phase 17

11. Relative Vastus Intermedius Force During the Concentric Phase 17

12. Relative Rectus Femoris Force During the Concentric Phase 18

13. Relative Gastrocnemius Force During the Concentric Phase 19

14. Relative Muscle Torques and Net Joint Moment at the Hip During Each of the Conditions 20

15. Contribution of Individual Muscles to the NJM at the Hip 21

16. Relative Muscle Torques of the Individual Hip Extensors at the Hip 22

17. Contribution of the Individual Hip Extensors to the NJM at the Hip 22

18. Relative Muscle Torque of the Rectus Femoris at the Hip 23

19. Contribution of the Rectus Femoris to the NJM at the Hip 23

20. Relative Muscle Torques and Net Joint Moment at the Knee 
21. Contribution of Individual Muscles to the NJM at the Knee

22. Relative Muscle Torques of the Individual Knee Flexors at the Knee

23. Contribution of the Individual Knee Flexors to the NJM at the Knee

24. Relative Muscle Torques of the Individual Knee Extensors at the Knee

25. Contribution of the Individual Knee Extensors to the NJM at the Knee

26. Relative Muscle Torques and Net Joint Moment at the Ankle

During Each of the Conditions

27. Contribution of Individual Muscles to the NJM at the Ankle

28. Relative Muscle Torques of the Individual Plantarflexors at the Ankle

29. Contribution of Individual Plantarflexors to the NJM at the Ankle 


\section{CHAPTER I: MODELING INDIVIDUAL MUSCLE FORCES DURING LOADED AND}

\section{UNLOADED HEXBAR VERTICAL JUMPS}

\section{Introduction}

Power and speed are key aspects of athletic performance and success in a multitude of sports (Swinton et al., 2012; T. S. Turner et al., 2015a, 2015b). Vertical jumping is one expression of lower body performance relevant to many sports and is often used in training for the development of athletic ability (Lees et al., 2004; Swinton et al., 2012). During human movement, including vertical jumping, biarticular muscles are of particular importance as they contribute in a different way than their uniarticular counterparts (Gregoire et al., 1984; Jacobs \& Bobbert, 1996; Nagano et al., 2005; Prilutsky \& Zatsiorsky, 1994; van Ingen Schenau et al., 1990; Zajac, 1993).

In both experimental and optimal control studies, results suggest uniarticular muscles are primarily responsible for propulsion, while the biarticular muscles finetune the movement (Nagano et al., 2005; Zajac, 1993) and transfer power distally (Prilutsky \& Zatsiorsky, 1994). In exploring the execution of maximal effort vertical jumps, muscle forces have been reported for both uniarticular and biarticular muscles, but only for the unloaded conditions (Cleather, 2019; Cleather et al., 2011; Cleather \& Cushion, 2019; Nagano et al., 2005; Pandy et al., 1990).

To improve an explosive movement such as a vertical jump, practicing that motion is important to be able to best utilize increased muscular strength (Bobbert \& Van Soest, 1994). Jump squat training is a method of training where the vertical jump is loaded (Lockie \& Lazar, 2017) in order to increase intensity of training, which allows for higher force generation and more power (Swinton et al., 2012). In such, jump squat training has been shown to improve 
variables related to athletic performance, such as jump height and sprint times (Mcbride et al., 2002).

Common methods of loading a vertical jump can vary, including a barbell across the shoulders (Mcbride et al., 2002; Swinton et al., 2012; A. P. Turner et al., 2012), a weighted vest (Feeney et al., 2016), or a hexagonal barbell (Swinton et al., 2012; T. S. Turner et al., 2015a, 2015b). The mode of loading affects the center of mass of the load on the body and thus can affect movement patterns (Swinton et al., 2012). Hexagonal barbell (hexbar) jump squats have the benefit of placing the load closer to the whole body center of mass and thus better mimicking normal jumping mechanics (Swinton et al., 2012), which is important in improving jump height (Bobbert \& Van Soest, 1994). In addition to variations in the mode of loading, the amount of loading can also vary (Feeney et al., 2016; Swinton et al., 2012; A. P. Turner et al., 2012; T. S. Turner et al., 2015a, 2015b), and the external load applied can alter kinematics and kinetics (Feeney et al., 2016; Kellis et al., 2005; Swinton et al., 2012; A. P. Turner et al., 2012; T. S. Turner et al., 2015b). With higher loads, a decreased depth of countermovement and reduced center of mass velocity have been reported (Feeney et al., 2016), as well as a reduced extension velocity of the joints and increased peak ground reaction force during propulsion (Kellis et al., 2005). Given that peak power generally occurs at lighter loads (Feeney et al., 2016; Swinton et al., 2012; A. P. Turner et al., 2012; T. S. Turner et al., 2015b), optimal loading of jump squats is suggested at loads of 10-20\% 1RM (Swinton et al., 2012; T. S. Turner et al., 2015b). How total body power, and the optimal load to achieve peak power, relates to individual muscle forces remains unclear. Although muscle forces across loading have not been investigated during a loaded jump squat, loading effects on muscle forces during traditional squats have been reported (Kipp et al., 2020a). 
When exploring individual muscle forces across loads during the squat, Kipp et al. (2020a) found peak force increased with load for the gluteus maximus, gluteus medius, and each vasti, while no differences were found for the rectus femoris, soleus, gastrocnemius, or any of the hamstrings muscles. The bi-articulate rectus femoris has been shown to undergo minimal length change during the squat motion (Robertson et al., 2008), thus the isometric contraction is indicative of the tendinous action and the transmission of energy across joints (Prilutsky \& Zatsiorsky, 1994). While Kipp et al. (2020a) controlled for speed, Kellis et al. (2005) instructed participants to perform the squat as fast as possible and reported both an increase in GRF and a decrease in extension velocity of the hip, knee, and ankle with added load. As described by the force-velocity relationship of skeletal muscle, a reduced velocity would allow for greater force development by the muscles crossing the joint (Hill, 1938); therefore, the addition of load may elicit significant increases in muscle force output as joint extension is slowed.

Individual muscle forces during an unloaded vertical jump have been reported by numerous authors (Cleather, 2019; Cleather et al., 2011; Cleather \& Cushion, 2019; Nagano et al., 2005; Pandy et al., 1990) and the effects of load on lower limb kinematics and kinetics have been documented using traditional inverse dynamics analyses (Feeney et al., 2016; Kellis et al., 2005; Swinton et al., 2012; A. P. Turner et al., 2012; T. S. Turner et al., 2015b). Studies have also investigated the effects of increasing external loads on individual muscle forces during traditional squats (Kipp et al., 2020a); yet, how the load affects individual muscle forces during maximal speed and effort jump squats remains unclear.

The purpose of this study is to describe the individual muscle forces that occur during maximal unloaded vertical jumps and explore how those may change with increasing loads 
during the hexbar jump squat. Based on the reported muscle forces during traditional squats, the force-velocity relationship, and the reviewed literature:

1) Hypothesis 1: heavier loads will elicit higher peak muscle forces in uniarticulate muscles;

2) Hypothesis 2: due to their biarticular nature, the rectus femoris and biceps femoris long head will not change in peak forces across loads.

To further explore the effect of load on individual muscles during hexbar jump squats, a secondary-qualitative analysis was performed where individual muscle torques were calculated to explore how peak muscle force may relate to changes in contribution to the net joint moment.

\section{Methods}

\section{Subjects}

Ten male, collegiate athletes (age: $20.4 \pm 2.41$ years; height: $1.85 \pm 0.057 \mathrm{~m}$; weight: $108.8 \pm 14.02 \mathrm{~kg}$ ) participated in the study. All subjects had a minimum of 2 years resistance training experience with performing the deadlift and countermovement jump under direct supervision of a certified strength and conditioning coach. Subjects were also required to be clear of injury to the lower extremity or spine within the last 2 years. Informed consent was obtained from each subject prior to participation. Approval of all procedures was provided by the Illinois State University Institutional Review Board (Maeda, 2018).

\section{Instrumentation}

A 33 retro-reflective marker set was placed on the lower extremity for motion capture. Markers were placed on the sacrum and bilaterally on the iliac crest, greater trochanter, medial and lateral epicondyle of the femur, medial and lateral malleoli, the calcaneus, and the head of the 1 st and 5th metatarsal. Marker quad- and triad-clusters were placed bilaterally on the lateral 
aspects of the thigh and shank, respectively. Three dimensional trajectories of markers were captured at $200 \mathrm{~Hz}$ with a 10-camera optical motion capture system (Vicon ${ }^{\circledR}$, Denver, CO, USA). Jumps were performed with the right foot entirely on the force plate (Advanced Mechanical Technology, Inc., Watertown, MA, USA) and ground reaction forces were captured at $1000 \mathrm{~Hz}$.

\section{Procedures}

\section{Data Collection}

Data collection was completed across two sessions separated by a minimum of $48 \mathrm{hr}$. Session one consisted of the completion of a survey regarding age, mass, height, resistance training history, estimated 1-repetition maximum (1RM), and injury history, as well as determination of hexbar deadlift 1RM. The hexbar deadlift was performed with technique previously outlined (Lockie \& Lazar, 2017). Loads for warmup sets and the initial testing set were determined based on the subject-reported estimated 1RM. The warmup prior to determination of 1RM was completed as follows: 10 repetitions with an unloaded $20 \mathrm{~kg}$ hexbar, 8 repetitions at $20 \%$ of the estimated $1 \mathrm{RM}, 5$ repetitions at $40 \%$ estimated $1 \mathrm{RM}, 5$ repetitions at $60 \%$ estimated 1RM. Following the warmup, load was increased to $80 \%$ estimated $1 \mathrm{RM}$ and the subject attempted to complete 5 repetitions. If the load was successfully lifted for 5 repetitions, weight was increased and a second attempt was made to find a 5RM. Subjects rested a minimum of 2 min $30 \mathrm{~s}$ between sets. Once the 5RM was established, 1RM was estimated using the Epley formula (Epley, 1985). The mean 1RM across all subjects was $216.6 \pm 10.9 \mathrm{~kg}$.

Session two consisted of data collection, with subjects performing hexbar jump squats at set percentages of the 1RM determined during session one. Subjects performed vertical jumps under four conditions: control ( $0 \% 1 \mathrm{RM}), 20 \% 1 \mathrm{RM}, 40 \% 1 \mathrm{RM}$, and $60 \% 1 \mathrm{RM}$. Prior to 
performing any dynamic data trials, a static calibration trial was captured. The control condition was performed first in all subjects, and the loaded conditions were subsequently completed in a randomized order to remove trial order bias. The control condition was performed with the hands on the hips to limit arm involvement in order to best compare to the loaded conditions. The weights used for loaded conditions were calculated using the determined 1RM. Five trials were performed for each condition, separated by a minimum of $2 \min 30 \mathrm{~s}$ rest. For each trial, subjects were instructed to stand with the right foot entirely on the force plate. Once in the standing position, subjects were instructed to perform a countermovement to a depth similar to that of an unweighted jump and then jump as high as possible while completing the motion as fast as possible. All 5 trials at each condition were utilized for analysis.

\section{Data Processing}

Ground reaction force (GRF) and 3D marker trajectory data were filtered in Vicon Nexus (Vicon ${ }^{\circledR}$, Denver, CO, USA) using a 4th order Butterworth filter at $300 \mathrm{~Hz}$ and $6 \mathrm{~Hz}$, respectively. Marker trajectories and GRF data were exported in CSV format and a customized MATLAB script (MatLab, The Mathworks, Inc., Natick, MA, USA) generated appropriate marker trajectory and GRF files for import into OpenSim v4.1 (Delp et al., 2007). Only the concentric phase of the jump was used for analysis and this was defined as the time from when the sacrum marker reached minima in the vertical direction to the time vertical GRF fell below 4 $\mathrm{N}$ at takeoff.

\section{Musculoskeletal Modeling}

The OpenSim model employed was Gait2354 (Anderson \& Pandy, 1999), which has 23 degrees of freedom driven by 54 hill-type musculotendon actuators. The metatarsophalangeal (MTP) and subtalar joint were both constrained during simulations (Hicks, 2018; Kipp \& Kim, 
2020). These joints were locked due to a lack of musculature in the model to control the respective degrees of freedom in the foot and ankle. The lumbar joint was also constrained during simulation because no tracking markers were placed on the upper body during motion capture. With these constraints, the model yielded joint angles and moments in all three planes at the hip (flexion/extension, adduction/abduction, internal/external rotation) and joint angles and moments in the sagittal plane at the knee (flexion/extension) and ankle (plantarflexion/dorsiflexion). Joint angles, joint moments, muscle forces, and muscle torques were calculated using OpenSim (Delp et al., 2007). Analysis with OpenSim was performed in a five-step process: scaling, inverse kinematics, inverse dynamics, static optimization, muscle analysis.

Scaling. Subject-specific scaling was performed using the static calibration trial, and dimensions of rigid bodies were scaled based on the relative distance between experimental markers as it compared to the distance between model markers (Delp et al., 2007). Scaling was adjusted until root mean square (RMS) was below $1 \mathrm{~cm}$ and maximum marker error was below 2 cm (Hicks, 2018). Four models were created for each subject, one for each condition, to account for the increasing mass of the hexbar. The control model had the mass of the subject, and each subsequent model had the additional mass of the loaded hexbar added to the mass of the model during scaling. Mass distribution was maintained during scaling for each subsequent model, and marker locations were identical between models. Because the original model was based on nonathletic population strength profiles, the maximal isometric force of muscles were doubled to account for the strength of this athletic population (Cleather et al., 2011; Tomescu et al., 2018).

Dynamic Analyses. Joint angles were calculated using the OpenSim inverse kinematics (IK) tool. In order to achieve optimal joint kinematics, marker tracking weights were adjusted to 
minimize RMS error and individual marker error at each time point (Lu \& O’Connor, 1999). The coordinates output from IK analysis were filtered at $13 \mathrm{~Hz}$ for all subsequent calculations. Joint moments were calculated using inverse dynamics (ID). Net joint moments were iteratively calculated by solving the equations of motion (Whittlesey \& Robertson, 2014). External loads were applied directly to the foot at the point of contact with the force plate (Delp et al., 2007).

Individual muscle forces were calculated using the OpenSim static optimization (SO) tool. Activation of muscles were bounded between 0 and 1 (Pandy et al., 1990; Roelker et al., 2020), and the objective function was set to minimize the sum of the squares of the individual muscle activations (Kipp et al., 2020a; Maniar et al., 2019). External loads applied were the same as those used during ID, and reserve actuators were appended to the model's force set. Each actuator corresponded with a unique degree of freedom within the model. Hence, for each trial, the optimal forces of the reserve actuators were systematically stepped down to minimum values while still allowing the static optimization tool to find a solution. The solution from SO includes muscle forces at each time point, while minimizing the objective function, which solve the equations of motion defined by kinematic input (Delp et al., 2007) obtained from IK.

The Muscle Analysis (MA) tool within OpenSim was used to calculate the moment arm of each muscle at each joint. The calculated moment arms were used to compute individual muscle torques by multiplying the moment arm and muscle force calculated with SO at each time point (Kipp et al., 2020b).

\section{Data Analysis}

Custom MATLAB scripts were used for data extraction from OpenSim output files. Joint angles and joint moment outputs were compared to those from Visual3D (Visual3D, C-Motion, Inc., Germantown, MD, USA) for qualitative validity. Peak muscle forces during the defined 
concentric phase of each trial were calculated for the following muscles: gluteus maximus (GMAX), biceps femoris - long head (BFL), rectus femoris (RF), vastus intermedius (VAST), gastrocnemius - medial head (GAS), soleus (SOL). The model contains three separate actuators representing GMAX, so the sum of these actuators was used. Peak muscle forces were calculated in both absolute terms and relative to body mass $(\mathrm{xBW})$. Mean peak muscle forces were calculated for each load and averaged across subjects.

Individual muscle torques were calculated as the product of the moment arm as calculated by MA and the muscle force as estimated with SO, computed at each time point. For GMAX, torques for each of the three actuators representing the GMAX were individually calculated and then summed to represent a single torque for the GMAX. Moment arms and muscle torques were averaged across trials for each condition. To calculate the individual contribution of each muscle to the net joint moment (NJM), the individual muscle torque was divided by the NJM calculated by ID and then multiplied by 100 . Biarticular muscles were assessed at both joints, and the SO results were used at full value at both joints.

All data were time normalized to 101 points for graphical purposes. Muscle force timeseries for the analyzed muscles, as well as timeseries of joint angles and moments, were averaged by load for each subject. Additionally, timeseries of individual muscle torques were averaged by load for each subject. All timeseries were then averaged across all subjects to yield aggregate time series data sets. The contribution of each muscle to the NJM was calculated with the group means.

\section{Statistical Analysis}

Statistical analyses were performed using SPSS software (version 24.0, SPSS Inc., Chicago, IL, USA). Significance was set a priori at $\mathrm{p}<0.05$. The dependent variables used for 
analyses were the mean relative peak muscle forces for GMAX, BFL, RF, VAST, GAS, and SOL within each condition, and the independent variable was load $(0 \%, 20 \%, 40 \%, 60 \%)$. A separate repeated-measures ANOVA (RMANOVA) was performed for each dependent variable to assess differences in relative peak muscle force across loads. Pair-wise comparisons utilizing least significant differences (LSD) were conducted where appropriate.

\section{Results}

Peak jump height as measured by peak vertical height of the sacrum marker above the ground is presented in Figure 1. Peak height achieved was reduced as load increased, by an average of $30 \mathrm{~cm}$ from control to $60 \%$.

\section{Figure 1}

Peak Height Above the Ground of the Sacrum Marker Across Loading Conditions

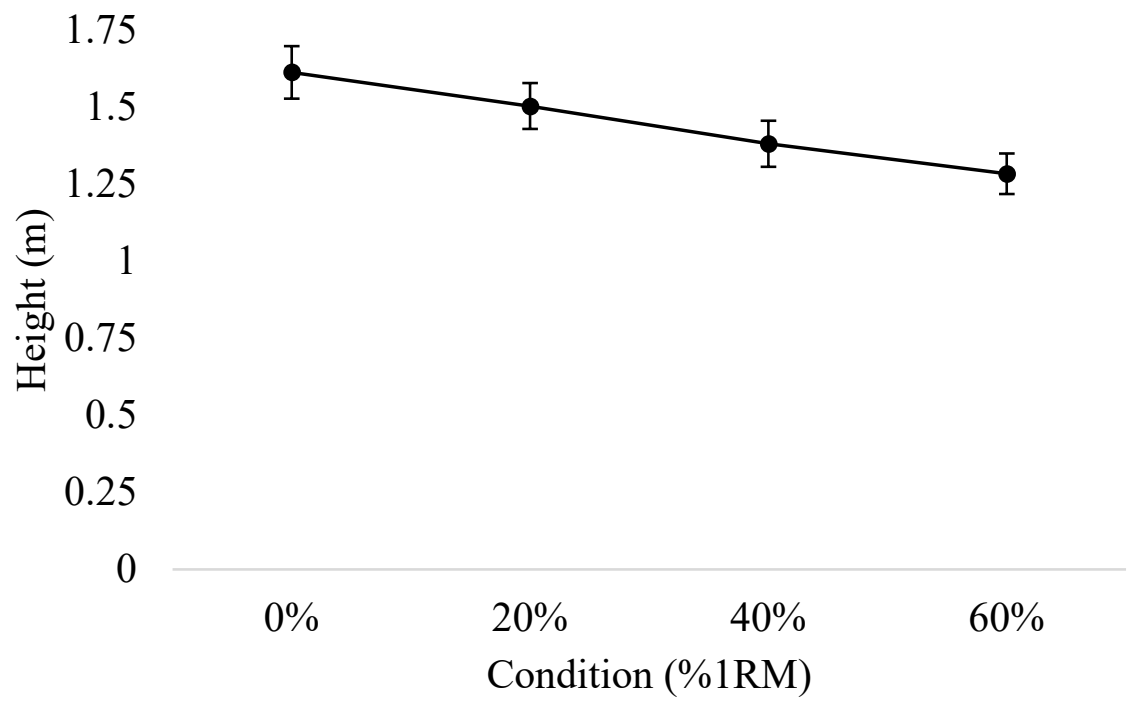

Timeseries of sagittal plane hip, knee and ankle joint angles across conditions are presented in Figures 2-4. Qualitatively, while the ankle angle did not show substantial changes, the knee and hip angles did differ between the unloaded and loaded conditions, with a more flexed position during the unloaded condition. 
Figure 2

Hip Joint Angle During the Concentric Phase

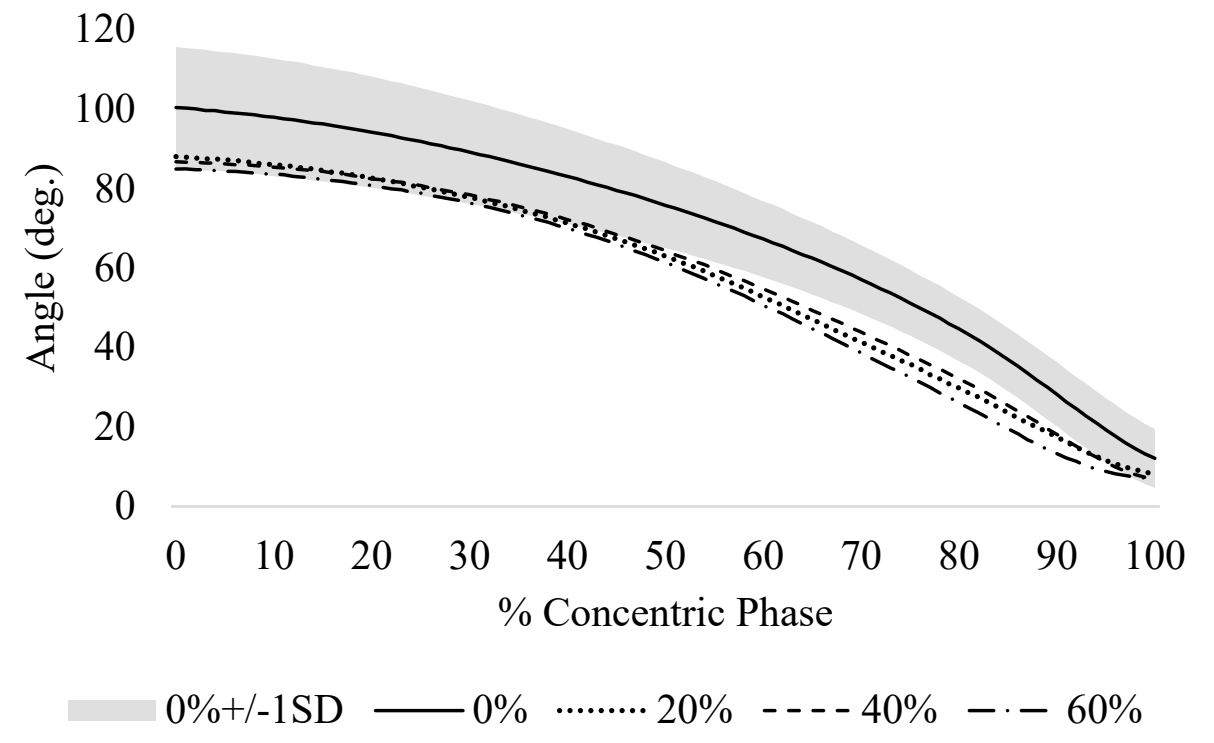

Note. Flexion is positive, extension is negative.

\section{Figure 3}

Knee Joint Angle During the Concentric Phase

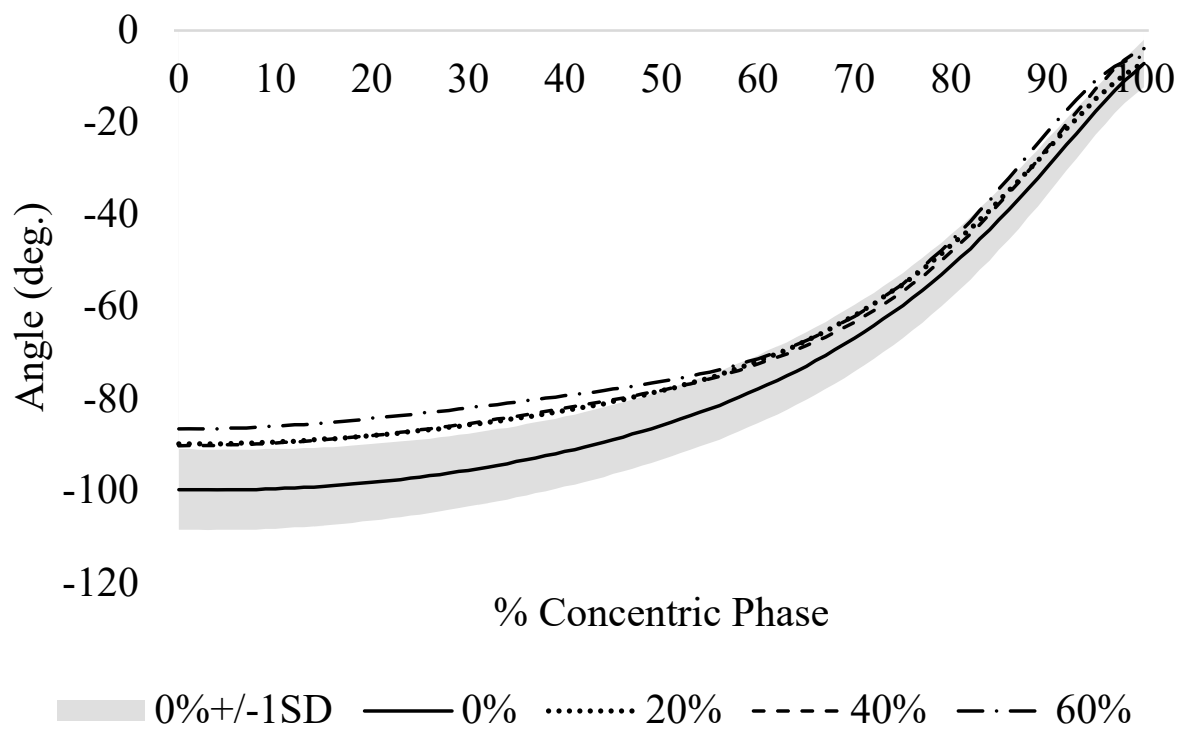


Note. Knee extension is positive, knee flexion is negative.

\section{Figure 4}

Ankle Joint Angle During the Concentric Phase

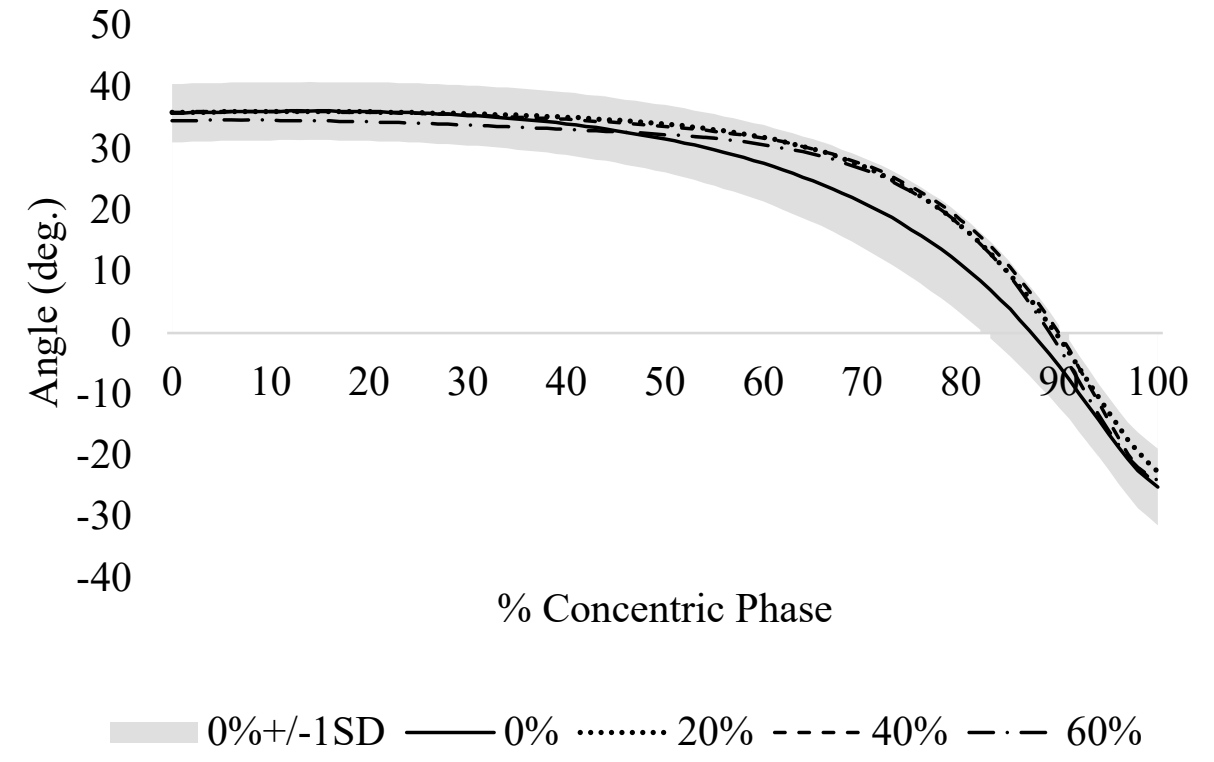

Note. Dorsiflexion is positive, plantarflexion is negative.

Sagittal plane NJM are presented in Figures 5-7. Qualitatively, differences in hip NJM were present for portions of the concentric phase, with the loaded conditions exhibiting higher magnitudes than the unloaded. More pronounced differences between conditions were noted for the knee NJM, with a greater extension moment as load increased. Lastly, the ankle NJM had substantial increases with increasing load, with clear differences between individual conditions. 


\section{Figure 5}

Relative Hip Net Joint Moment During the Concentric Phase

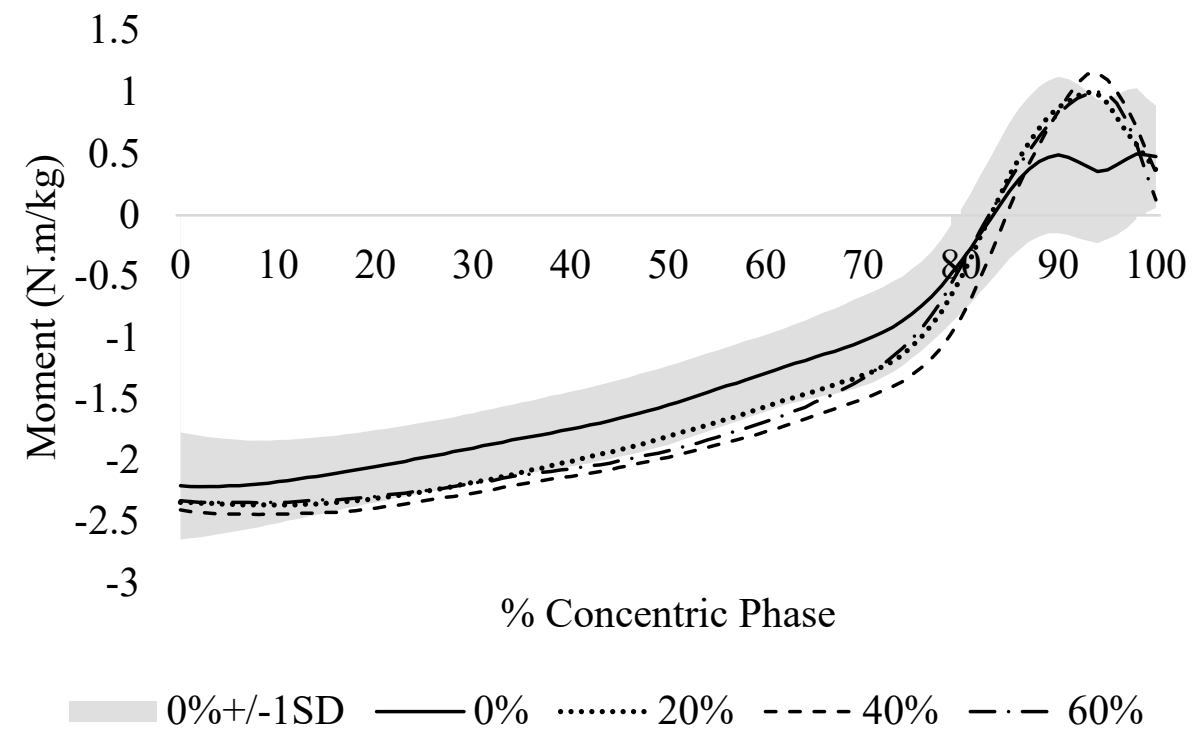

Note. Internal extension moment is negative, flexion moment is positive.

\section{Figure 6}

Relative Knee Net Joint Moment During the Concentric Phase

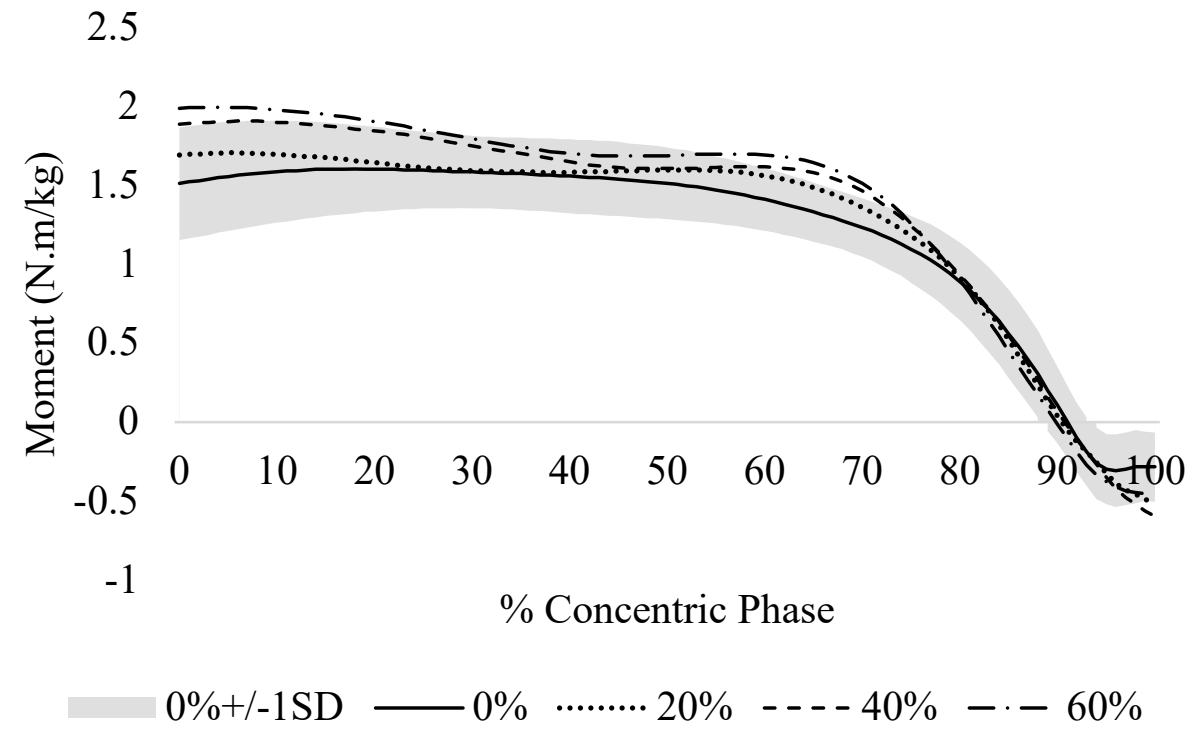


Note. Internal extension moment is positive, flexion moment is negative.

\section{Figure 7}

Relative Ankle Net Joint Moment During the Concentric Phase

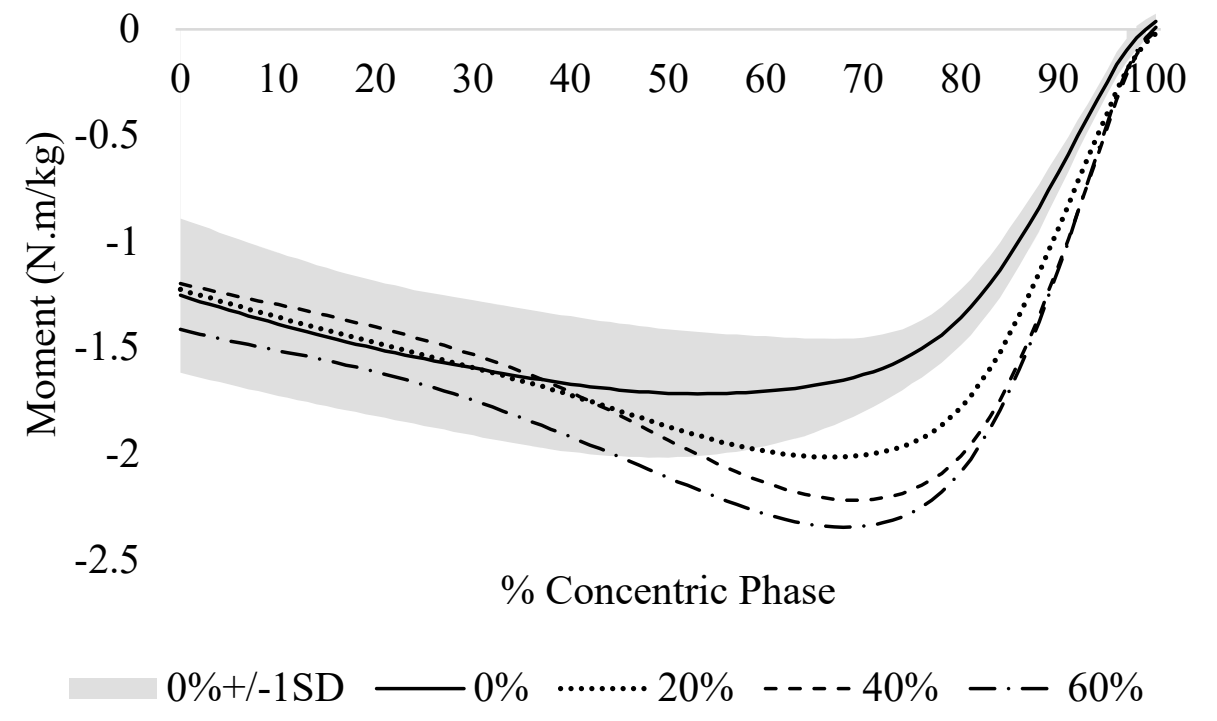

Note. Internal extension moment is negative, flexion moment is positive.

\section{Individual Muscle Forces}

There were no significant differences in peak muscle force across loads for GMAX ( $\mathrm{p}=$ 0.325; Table 1, Figure 8), BFL ( $\mathrm{p}=0.369$; Table 1, Figure 9), or SOL $(\mathrm{p}=0.122$; Table 1, Figure 10). 
Table 1

Relative Peak Muscle Forces Across Loads

\begin{tabular}{|c|c|c|c|c|}
\hline \multirow[b]{2}{*}{ Muscle } & \multicolumn{4}{|c|}{ Load condition (\%1RM) } \\
\hline & Control $(0 \%)$ & $20 \%$ & $40 \%$ & $60 \%$ \\
\hline \multirow[t]{2}{*}{ GMAX } & $2.53 \pm 0.18$ & $2.60 \pm 0.20$ & $2.62 \pm 0.17$ & $2.41 \pm 0.18$ \\
\hline & $(2.12,2.94)$ & $(2.16,3.04)$ & $(2.25,3.00)$ & $(2.01,2.82)$ \\
\hline \multirow[t]{2}{*}{ VAST } & $7.89 \pm 0.24^{c, d}$ & $8.22 \pm 0.28^{d}$ & $8.47 \pm 0.30^{a}$ & $8.64 \pm 0.33^{a, b}$ \\
\hline & $(7.35,8.43)$ & $(7.58,8.86)$ & $(7.80,9.15)$ & $(7.90,9.38)$ \\
\hline \multirow[t]{2}{*}{ GAS } & $2.14 \pm 0.10^{b, c, d}$ & $2.47 \pm 0.14^{\mathrm{a}, \mathrm{c}, \mathrm{d}}$ & $2.72 \pm 0.12^{a, b, d}$ & $2.85 \pm 0.14^{a, b, c}$ \\
\hline & $(1.92,2.38)$ & $(2.17,2.78)$ & $(2.44,3.00)$ & $(2.54,3.17)$ \\
\hline \multirow[t]{2}{*}{ SOL } & $3.89 \pm 0.22$ & $4.02 \pm 0.19$ & $4.05 \pm 0.16$ & $4.27 \pm 0.18$ \\
\hline & $(3.40,4.38)$ & $(3.59,4.45)$ & $(3.68,4.42)$ & $(3.85,4.68)$ \\
\hline \multirow[t]{2}{*}{$\mathrm{RF}$} & $2.50 \pm 0.13^{b, c, d}$ & $2.32 \pm 0.17^{\mathrm{a}, \mathrm{d}}$ & $2.18 \pm 0.11^{a}$ & $1.98 \pm 0.20$ a,b \\
\hline & $(2.21,2.79)$ & $(1.93,2.71)$ & $(1.93,2.42)$ & $(1.53,2.42)$ \\
\hline \multirow[t]{2}{*}{ BFL } & $3.71 \pm 0.21$ & $3.61 \pm 0.22$ & $3.81 \pm 0.27$ & $3.56 \pm 0.29$ \\
\hline & $(3.24,4.18)$ & $(3.12,4.10)$ & $(3.21,4.41)$ & $(2.91,4.22)$ \\
\hline
\end{tabular}

Note: Relative forces are normalized to body weight $(\mathrm{xBW})$. Data is presented as Mean \pm SD (95\% CI lower bound, upper bound).

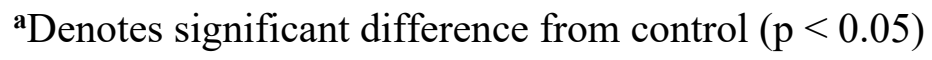

${ }^{\mathbf{b}}$ Denotes significant difference from $20 \%(\mathrm{p}<0.05)$

${ }^{\mathrm{c}}$ Denotes significant difference from $40 \%(\mathrm{p}<0.05)$

${ }^{d}$ Denotes significant difference from $60 \%(p<0.05)$ 


\section{Figure 8}

Relative Gluteus Maximum Force During the Concentric Phase

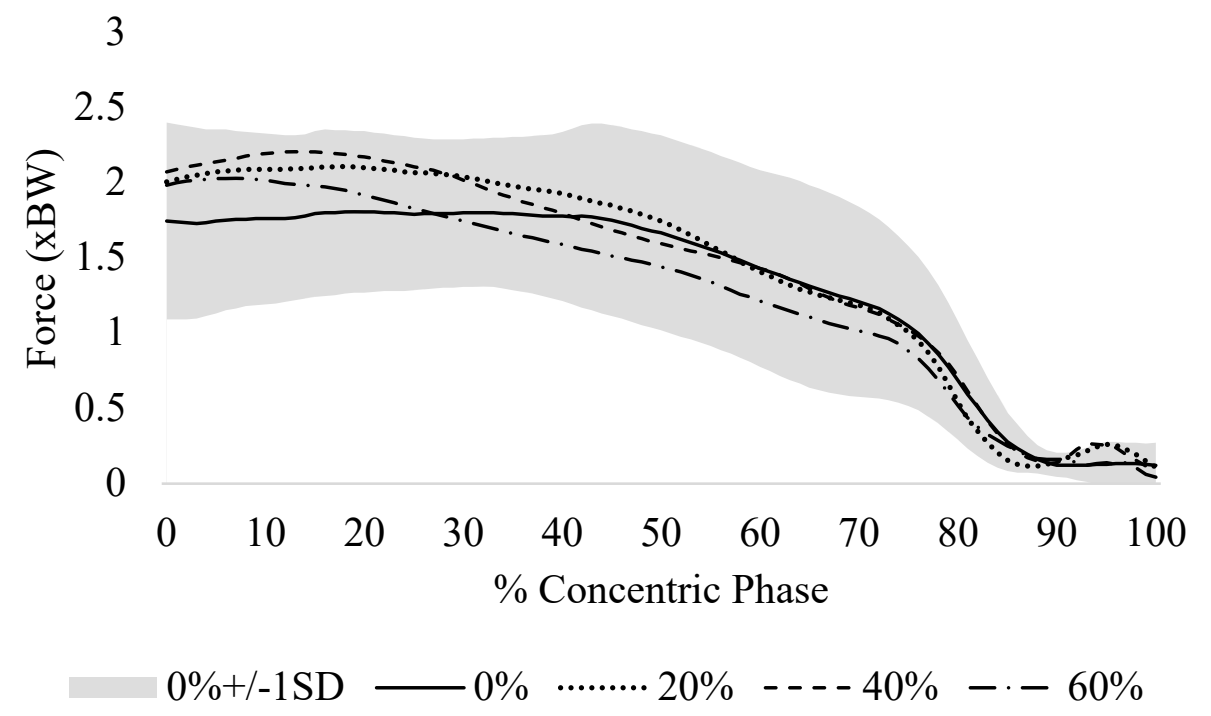

\section{Figure 9}

Relative Biceps Femoris - Long Head Force During the Concentric Phase

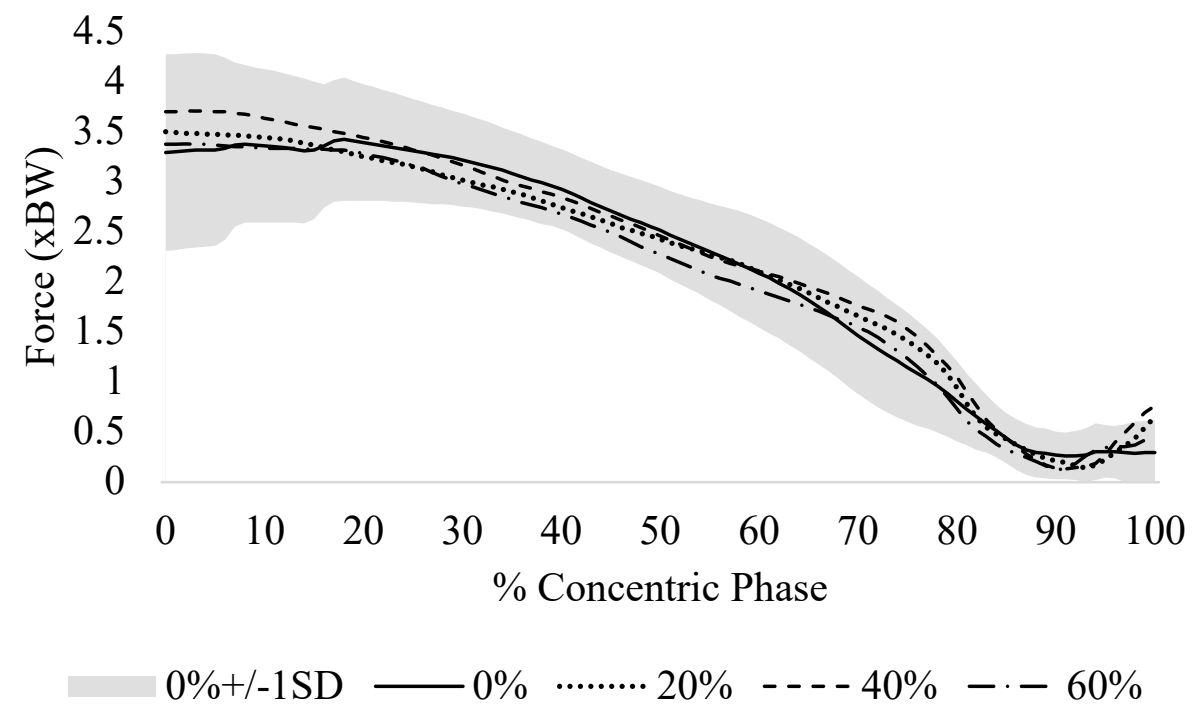




\section{Figure 10}

Relative Soleus Force During the Concentric Phase

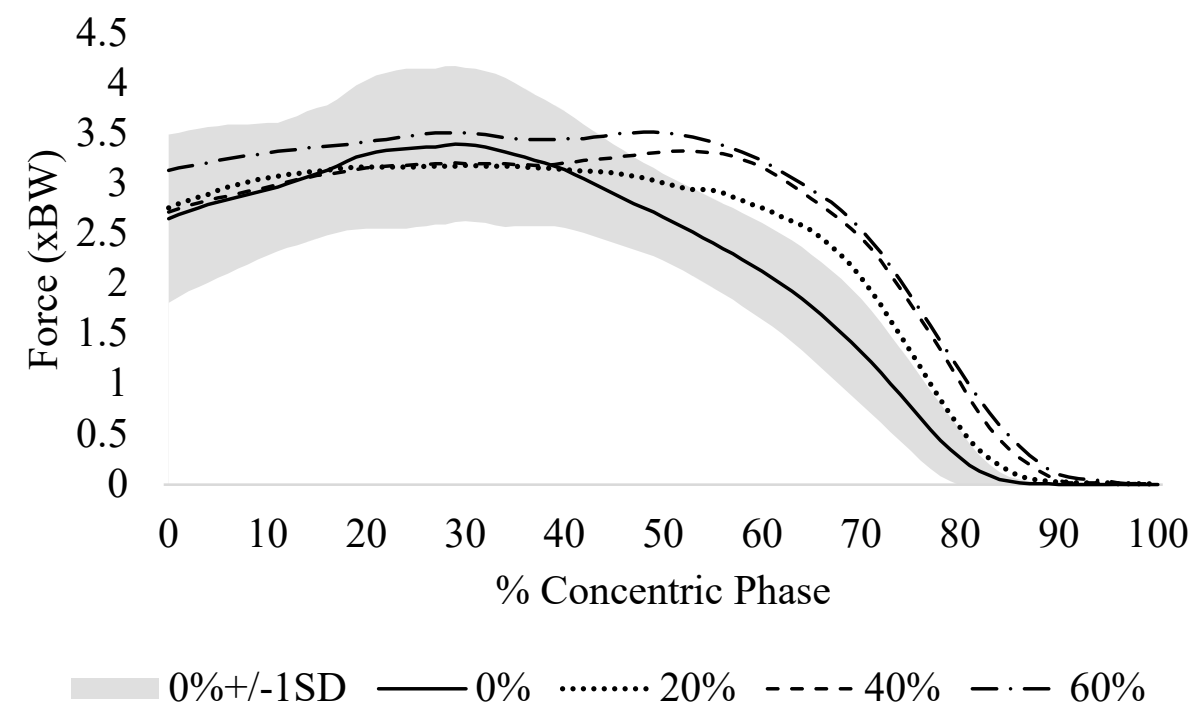

A significant increase in peak muscle force across loads existed for VAST $(p=0.009$;

Table 1, Figure 11), with significant differences between control and 40\% $(p=0.009)$, control and $60 \%(\mathrm{p}=0.015)$, and $20 \%$ and $60 \%(\mathrm{p}=0.011)$.

\section{Figure 11}

Relative Vastus Intermedius Force During the Concentric Phase

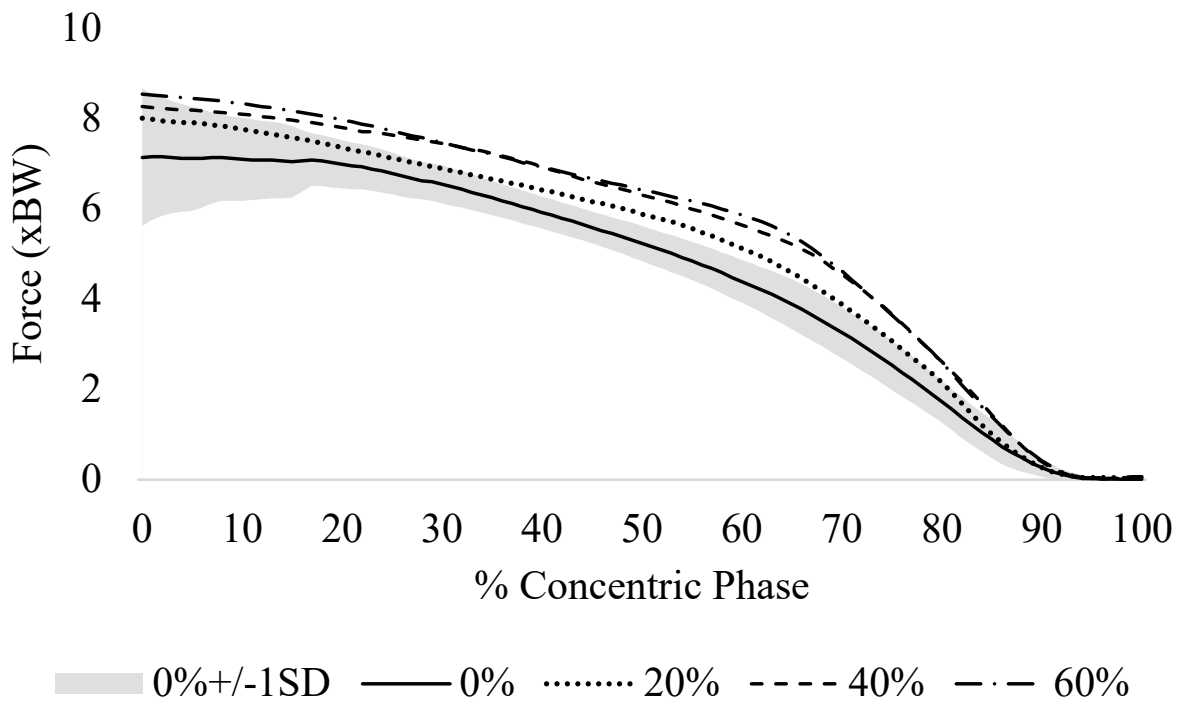


Significant decreases were noted for RF ( $p=0.017$; Table 1, Figure 12), with significant differences between control and 20\% $(p=0.034)$, control and $40 \%(p=0.037)$, control and $60 \%$ $(\mathrm{p}=0.005)$, and $20 \%$ and $60 \%(\mathrm{p}=0.032)$.

\section{Figure 12}

\section{Relative Rectus Femoris Force During the Concentric Phase}

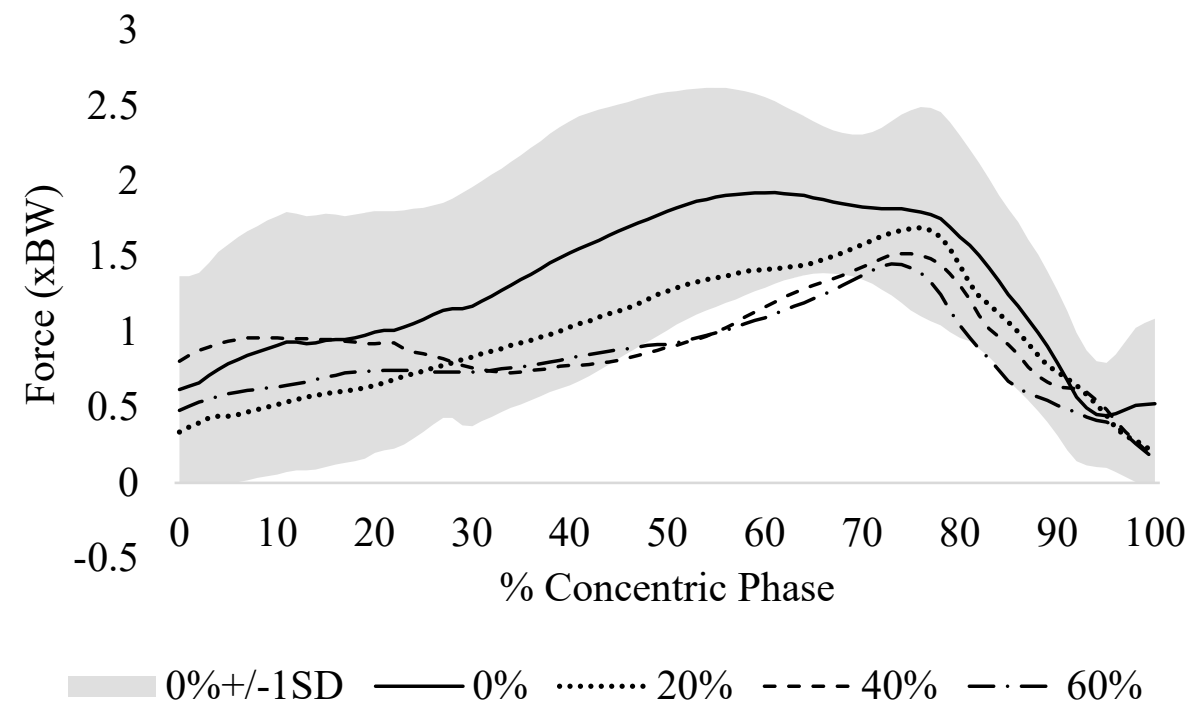

Lastly, GAS had significant differences across loads $(\mathrm{p}<0.001$; Table 1, Figure 13), with significant increases from control to each of the loaded conditions (all $p \leq 0.001$ ), between $20 \%$ and heavier conditions (all $\mathrm{p}<0.001)$, and between $40 \%$ and $60 \%(\mathrm{p}=0.038)$. Absolute peak force values are presented in Table 2 . 


\section{Figure 13}

Relative Gastrocnemius Force During the Concentric Phase

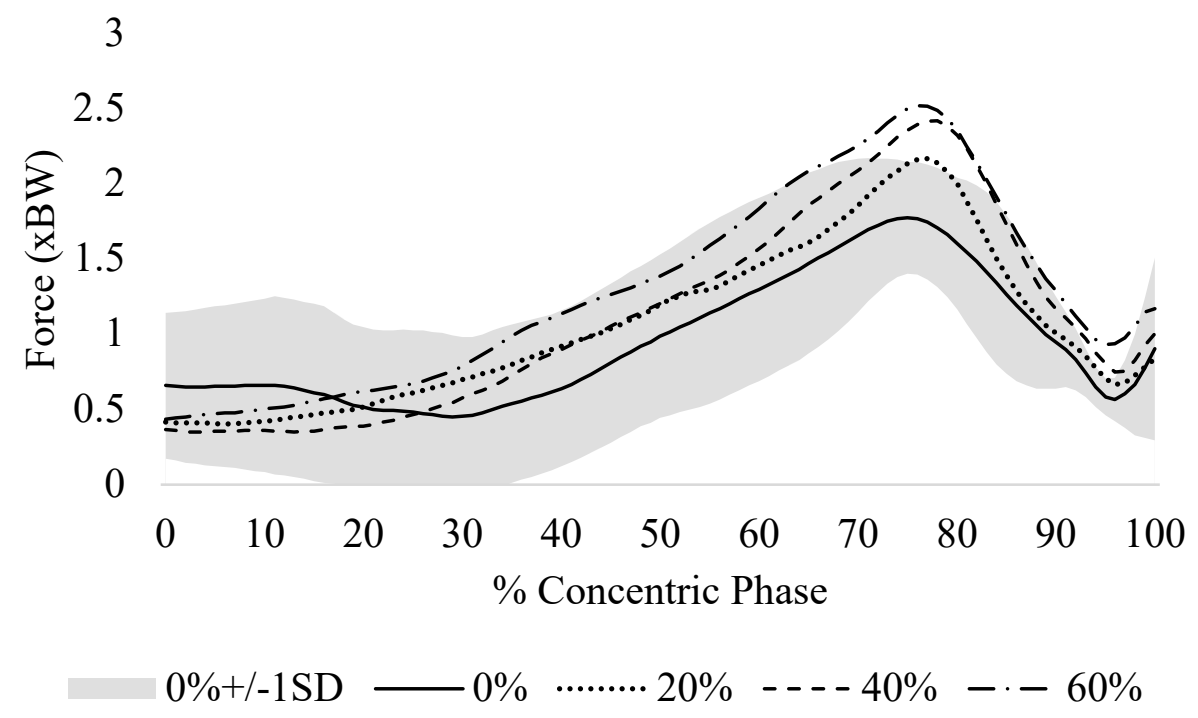

\section{Table 2}

Absolute Peak Muscle Forces Across Loads

\begin{tabular}{lcccc}
\hline & \multicolumn{4}{c}{ Load condition (\%1RM) } \\
\cline { 2 - 5 } Muscle & Control $(0 \%)$ & $20 \%$ & $40 \%$ & $60 \%$ \\
\hline GMAX & $2719.19 \pm 701.80$ & $2753.98 \pm 719.47$ & $2810.52 \pm 694.35$ & $2557.80 \pm 696.85$ \\
VAST & $8352.92 \pm 884.14$ & $8656.59 \pm 691.22$ & $8927.62 \pm 543.76$ & $9087.16 \pm 433.57$ \\
GAS & $2262.16 \pm 429.47$ & $2613.43 \pm 319.72$ & $2862.31 \pm 327.00$ & $3015.59 \pm 465.97$ \\
SOL & $4137.77 \pm 771.55$ & $4267.82 \pm 842.99$ & $4323.66 \pm 783.24$ & $4518.91 \pm 795.92$ \\
RF & $2671.47 \pm 528.48$ & $2481.84 \pm 697.95$ & $2320.20 \pm 490.38$ & $2128.93 \pm 738.50$ \\
BFL & $3888.65 \pm 417.41$ & $3802.04 \pm 307.20$ & $3979.79 \pm 528.88$ & $3712.39 \pm 628.25$ \\
\hline
\end{tabular}

Note: Absolute peak forces are reported in $\mathrm{N}$.

\section{Individual Muscle Torques and Contributions}

The hip NJM increased in magnitude with the addition of external load, but with minimal change between loaded conditions. The knee NJM also exhibited increases with additional load, 
although often not outside of one standard deviation from the control. Lastly, the ankle NJM increased in magnitude with increases in load, particularly at the point of peak NJM.

\section{Hip}

Timeseries of the individual muscle torques and NJM at the hip for each condition are presented in Figure 14, and the calculated contribution of each muscle to the NJM is presented in Figure 15.

\section{Figure 14}

\section{Relative Muscle Torques and Net Joint Moment at the Hip During Each of the Conditions}

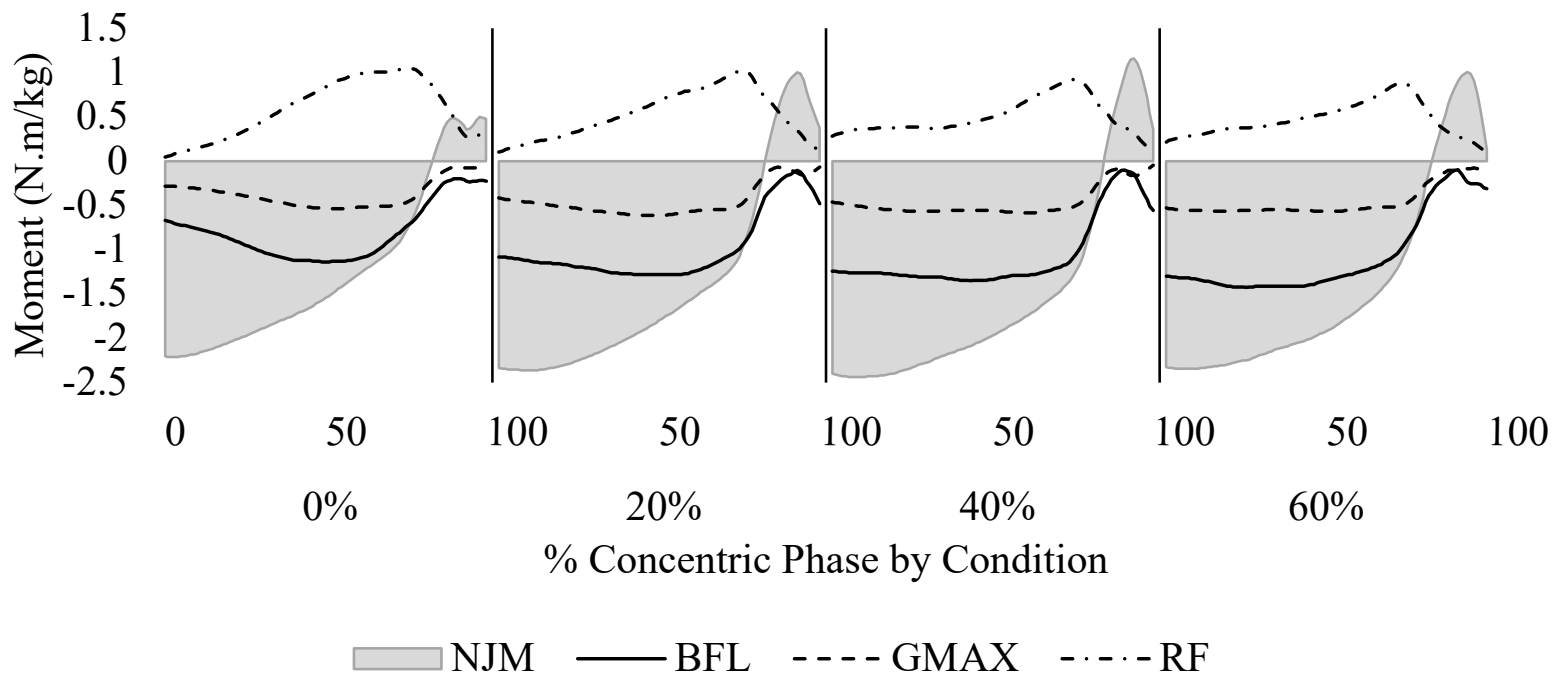

Note. NJM is shaded to designate net flexor (positive) versus net extensor (negative) patterns of the hip joint moment and represents a sum of all individual muscle torques ( $\mathrm{n}=14$ muscles). Only the BFL, RF, and GMAX are individually graphed due to being the largest muscle torques. 


\section{Figure 15}

Contribution of Individual Muscles to the NJM at the Hip

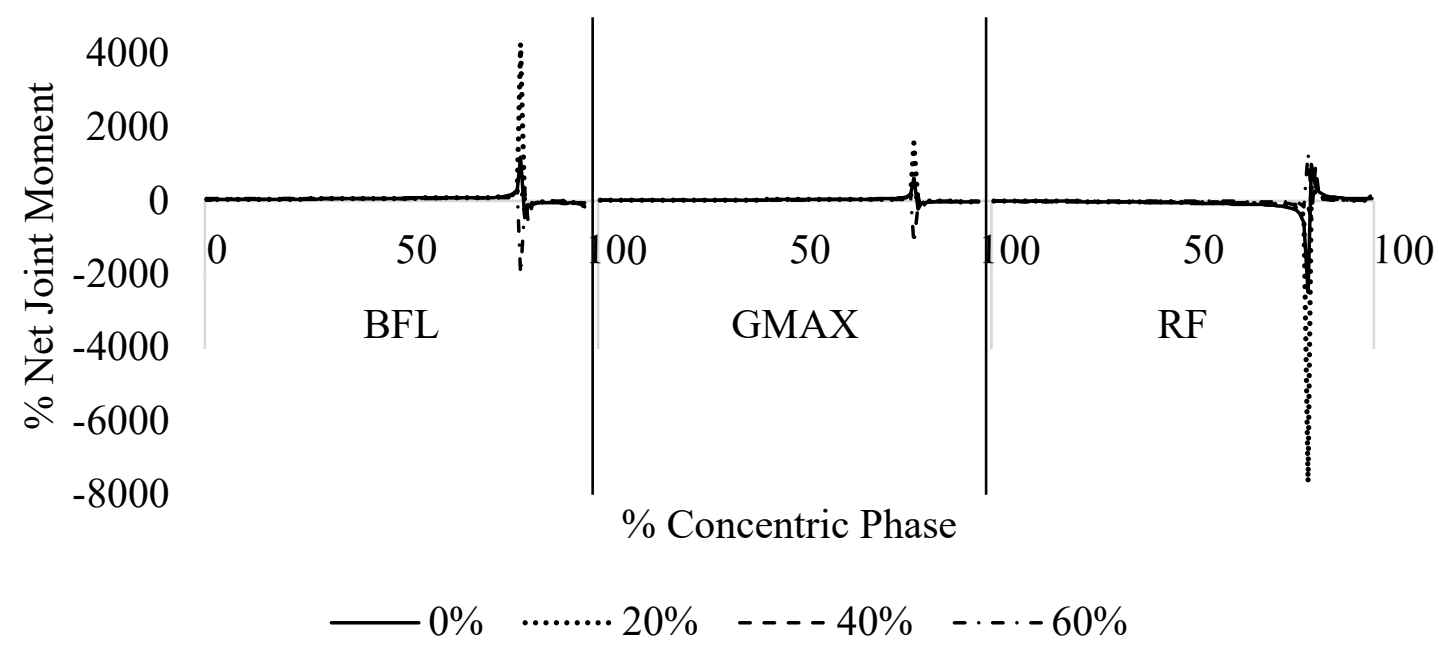

Note. Contribution was calculated as (individual muscle torque / NJM) * 100 .

Qualitatively, both hip extensors showed an increase in torque with the addition of external load, but not substantial increases between loads. The gluteus maximus and the biceps femoris - long head torques exhibited a similar timeseries in regard to shape, but the gluteus maximus values (Figure 16) were roughly half of the biceps femoris - long head values (Figure 16). As a result of this difference in torque, the contribution of each muscle to the NJM was similarly scaled. For the first $30 \%$ of the concentric phase, both hip extensors had increasing contributions as the load increased, but beyond that point there is no clear trend (Figure 17). In addition to the extensor torques, the rectus femoris creates a flexion torque about the hip. Similar to the force values for the rectus femoris, the peak torque was reduced with increasing loads (Figure 18). The combination of a reduced magnitude of the flexor torque and an increased magnitude in the NJM resulted in a substantial decrease in negative contribution from the rectus femoris as load increased (Figure 19). 


\section{Figure 16}

Relative Muscle Torques of the Individual Hip Extensors at the Hip

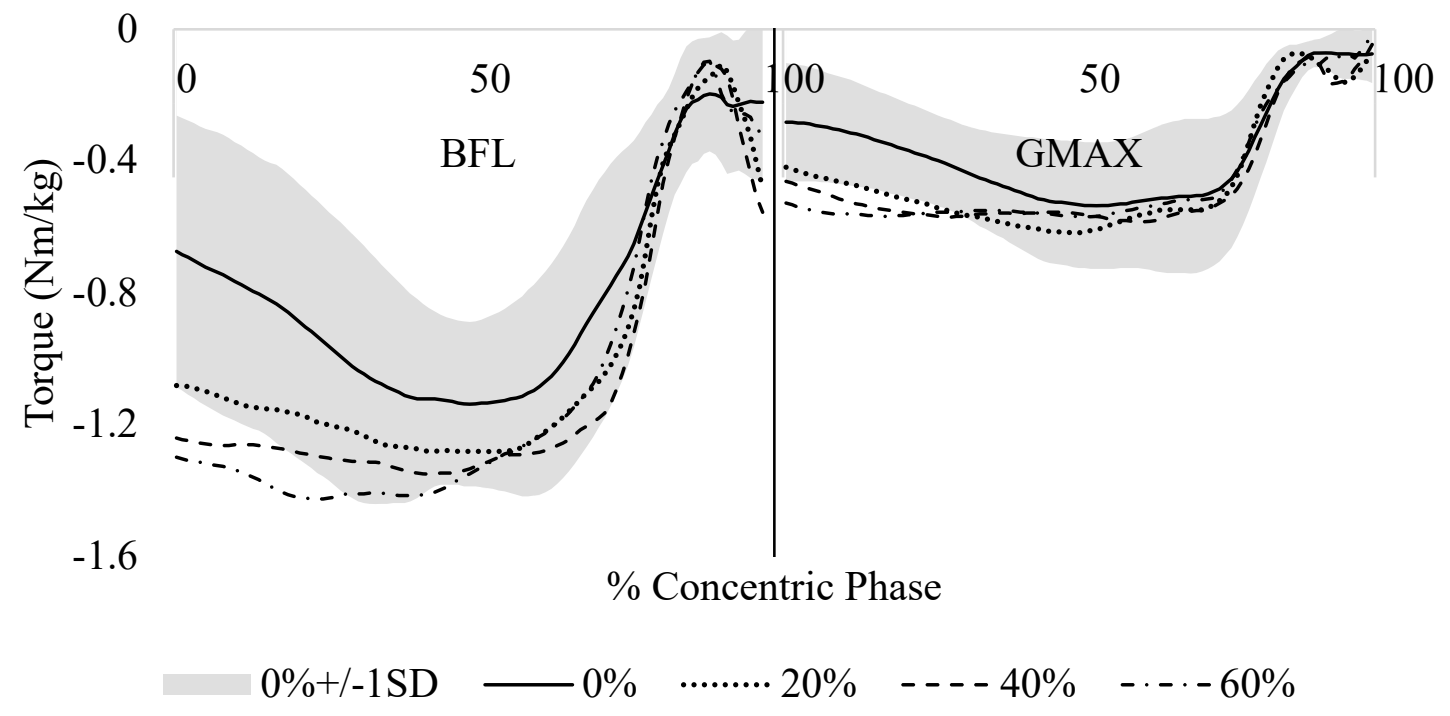

\section{Figure 17}

Contribution of the Individual Hip Extensors to the NJM at the Hip

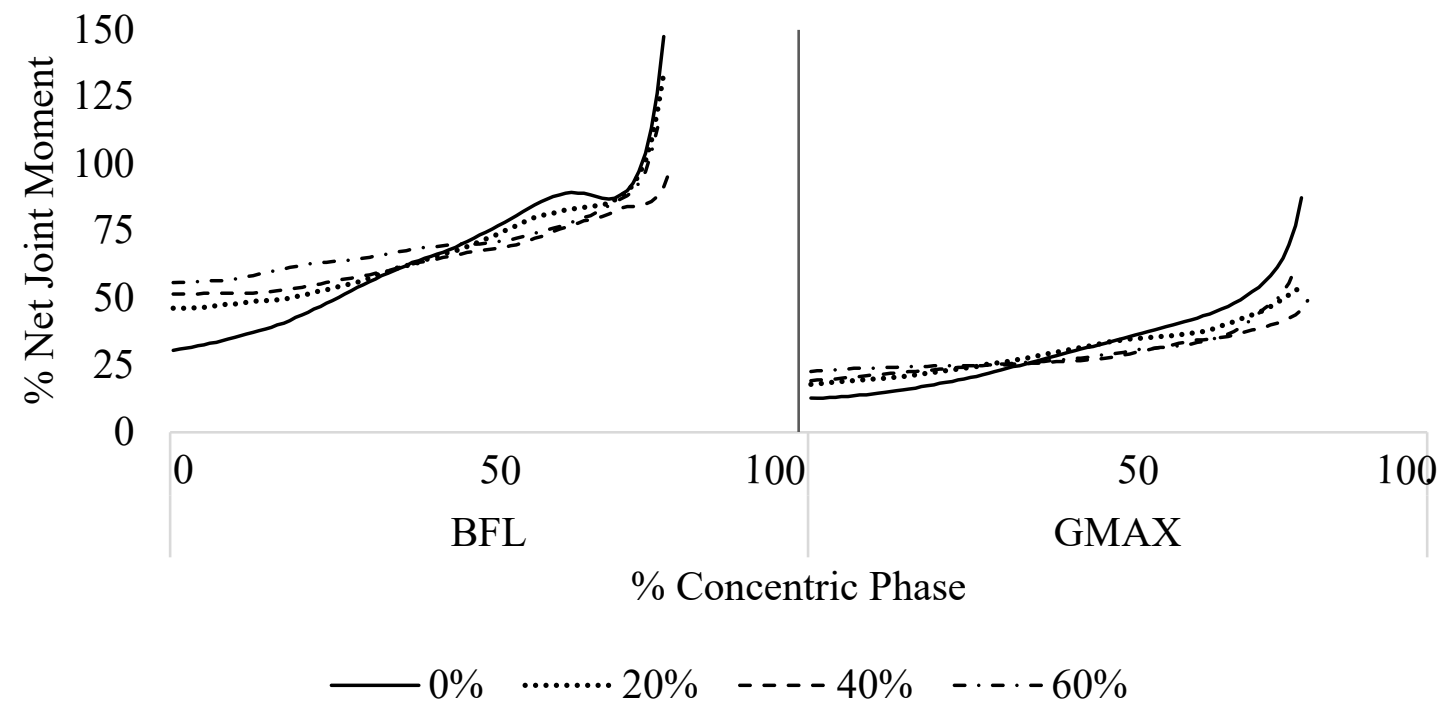

Note. Contribution was calculated as (individual muscle torque / NJM) * 100 . 


\section{Figure 18}

Relative Muscle Torque of the Rectus Femoris at the Hip

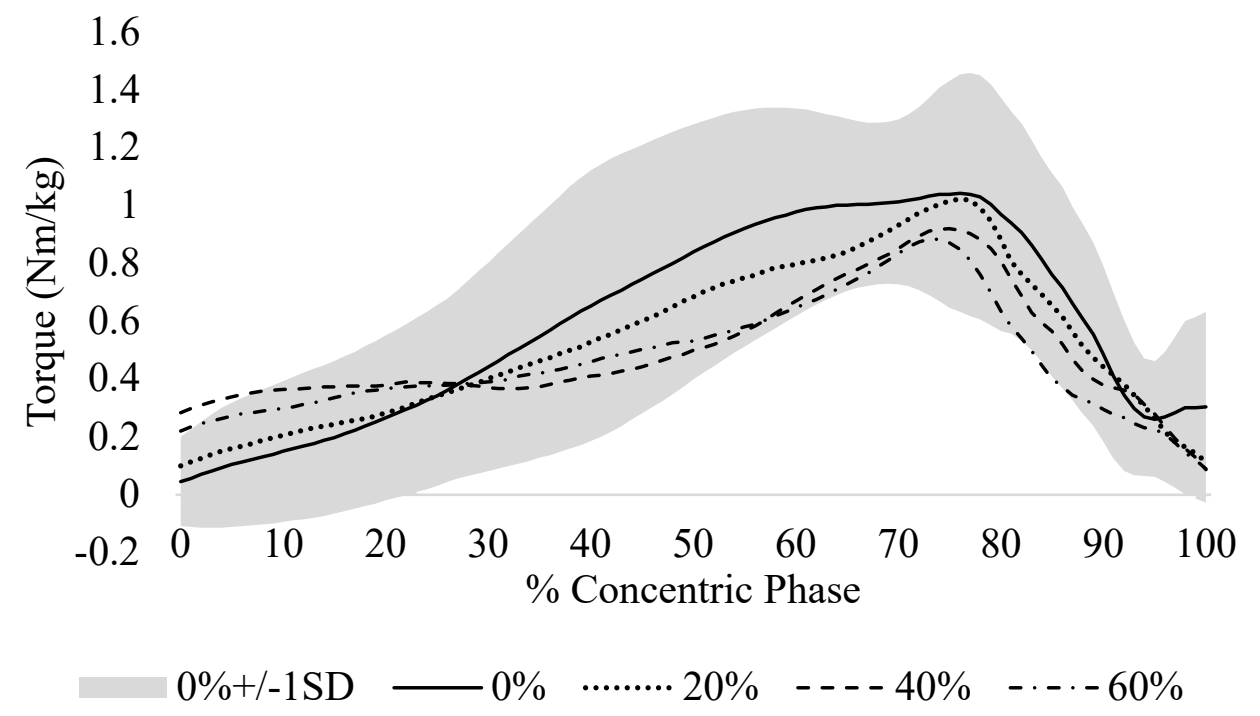

\section{Figure 19}

Contribution of the Rectus Femoris to the NJM at the Hip

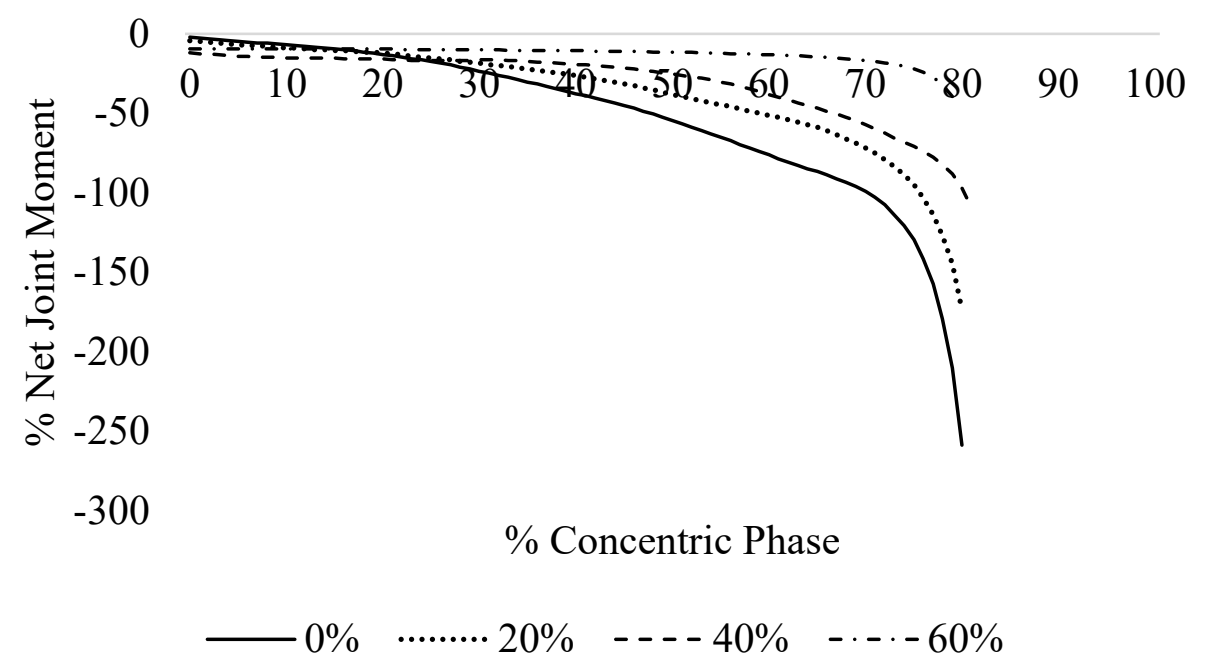

Note. Contribution was calculated as (individual muscle torque / NJM) * 100. 


\section{Knee}

Timeseries of the individual muscle torques and NJM at the knee for each condition are presented in Figure 20, and the calculated contribution of each muscle to the NJM is presented in Figure 21.

Figure 20

Relative Muscle Torques and Net Joint Moment at the Knee During Each of the Conditions

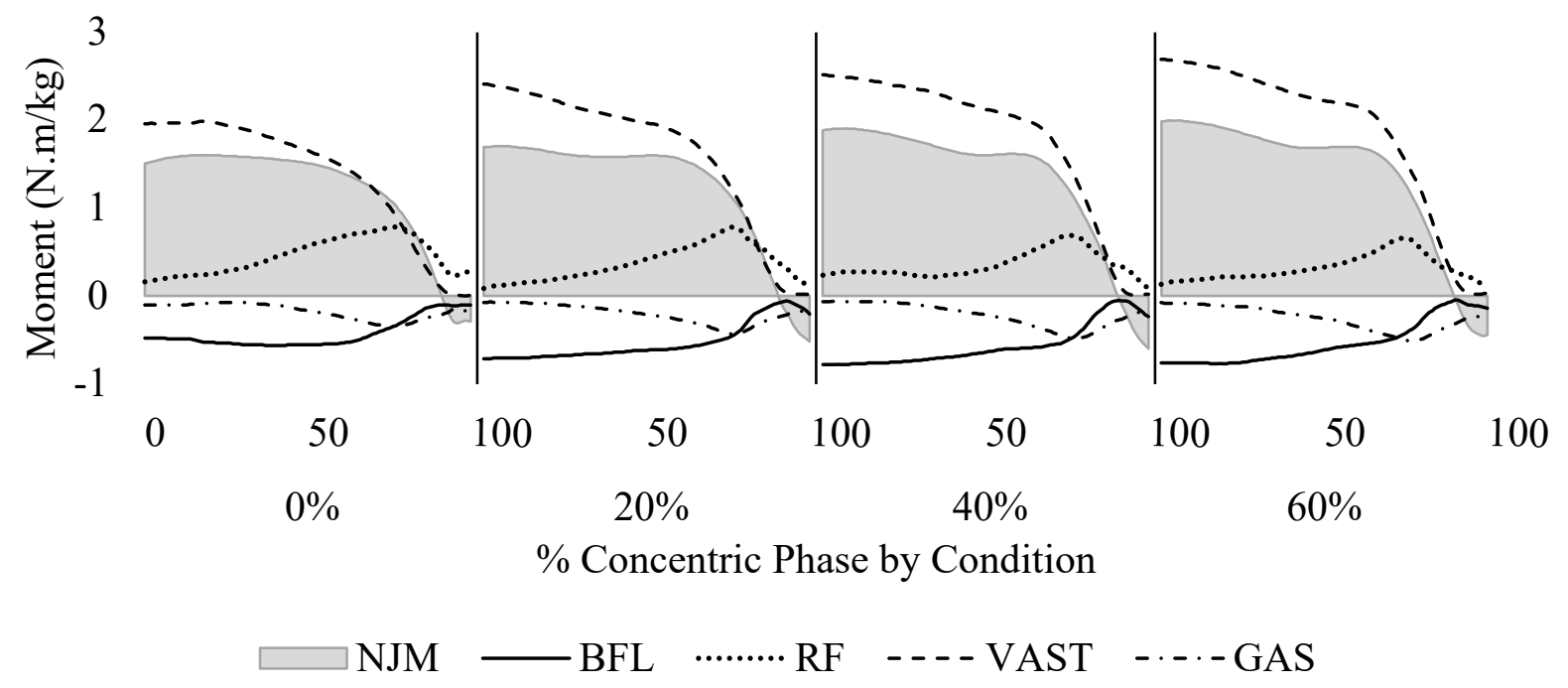

Note. NJM is shaded to designate net extensor (positive) versus net flexor (negative) patterns of the knee joint moment and represents a sum of all individual muscle torques ( $\mathrm{n}=8$ muscles). Only the BFL, RF, VAST, and GAS are individually graphed due to being the largest muscle torques. 


\section{Figure 21}

Contribution of Individual Muscles to the NJM at the Knee

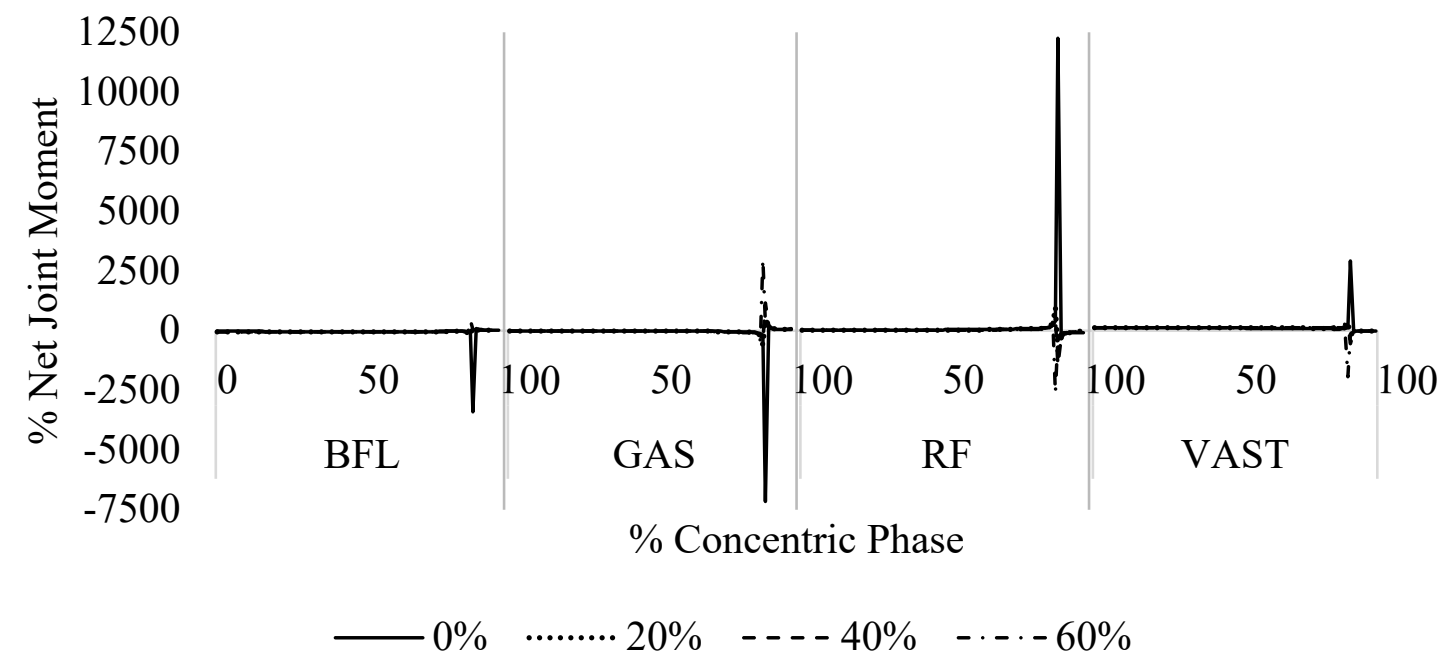

Note. Contribution was calculated as (individual muscle torque / NJM) $* 100$.

The gastrocnemius moment at the knee, while showing clear differences between loads, had a maximum difference of only $0.2 \mathrm{Nm} . \mathrm{kg}$ (Figure 22). The biceps femoris - long head had slightly larger differences in muscle torques at the beginning of the movement $(0.3 \mathrm{Nm} / \mathrm{kg})$, but any differences diminished as the movement was completed (Figure 22). The negative contribution of the gastrocnemius was consistent across loads, and the contribution of the biceps femoris - long head was relatively consistent for the duration of the concentric phase, remaining primarily between $-30 \%$ and $-40 \%$, and no clear trend as to differences between loads (Figure 23). The flexor torque generated by these two muscles showed a similar trend to the torque generated by the biceps femoris - long head where the control condition was less, but no clear difference existed between loaded conditions. The summed flexor torque created represents between a $-40 \%$ and $-60 \%$ contribution to the NJM for the majority of the movement, and then steadily increased to $-120 \%$ as the extensor NJM reached a minimum. 


\section{Figure 22}

Relative Muscle Torques of the Individual Knee Flexors at the Knee

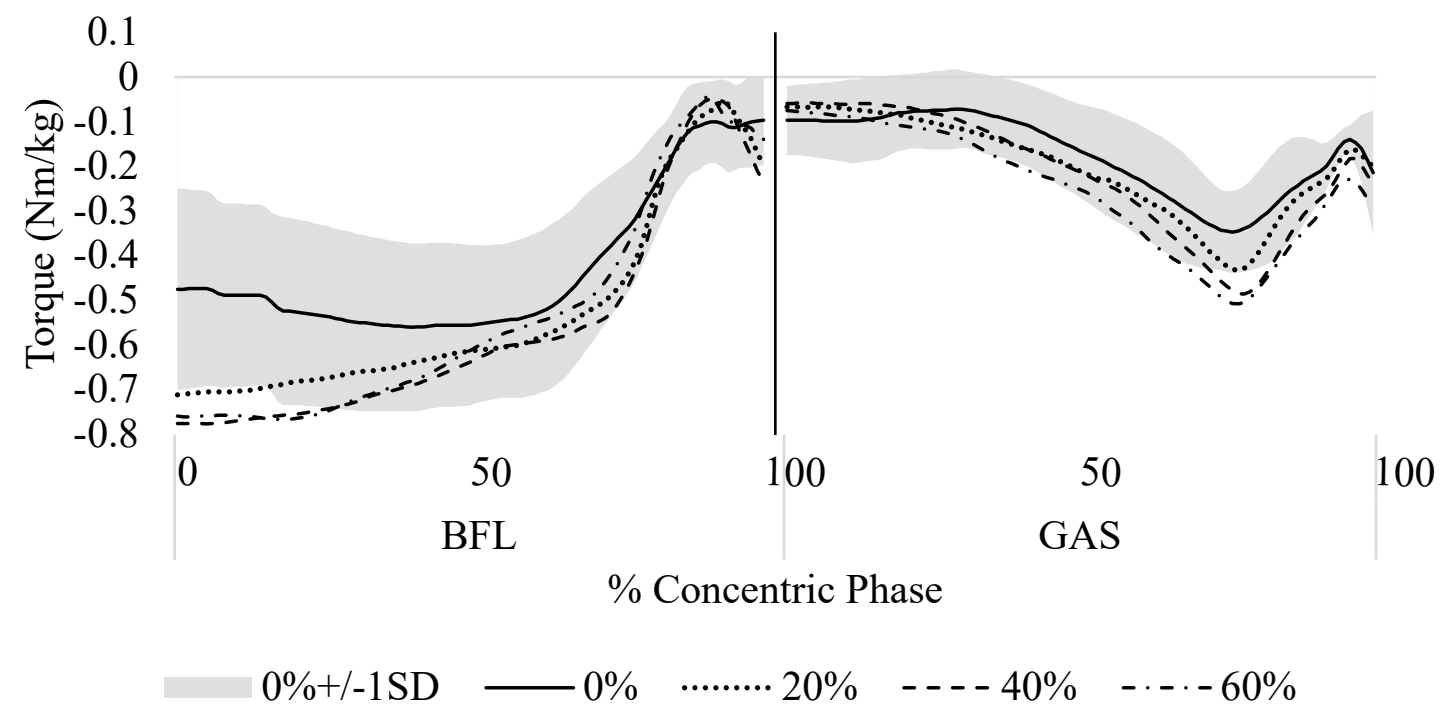

\section{Figure 23}

Contribution of the Individual Knee Flexors to the NJM at the Knee

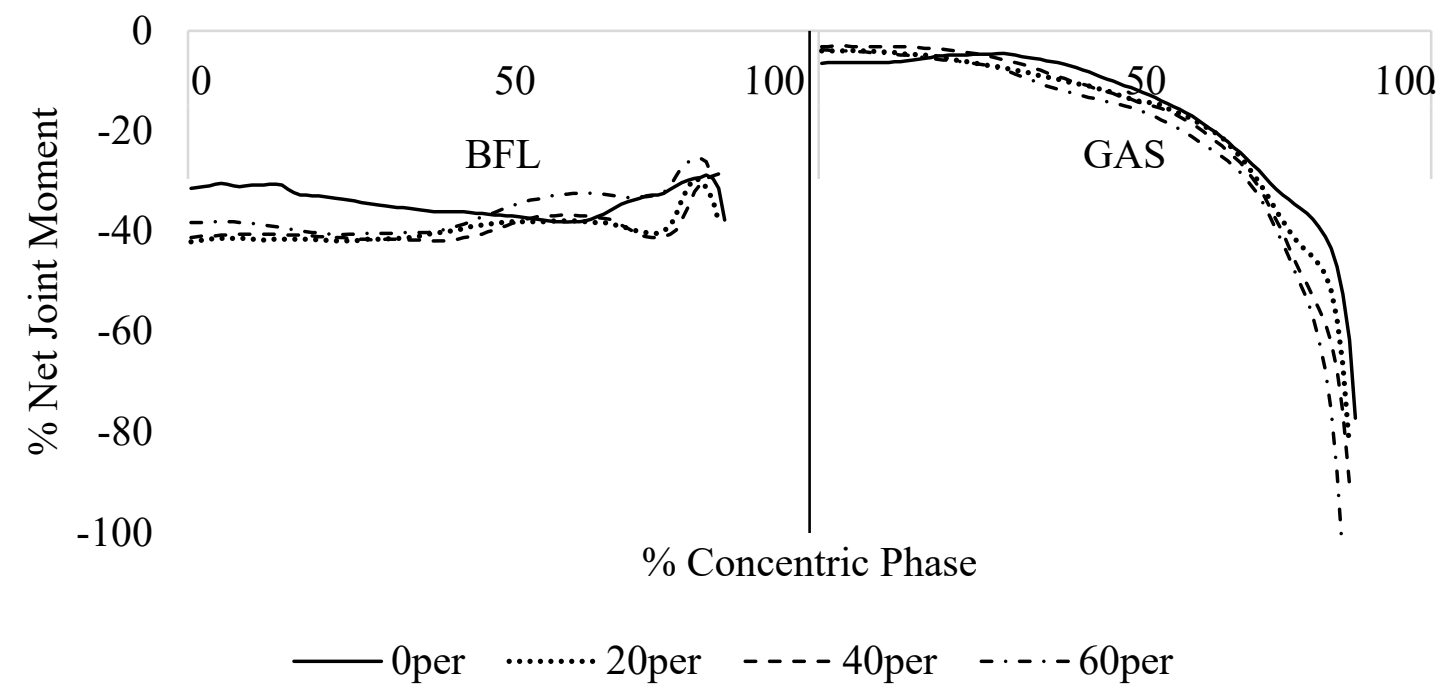

Note. Contribution was calculated as (individual muscle torque / NJM) * 100.

While the knee flexors created a negative contribution to the NJM, the quadriceps created a considerably larger torque. Just as the peak rectus femoris muscle force decreased with 
increasing load, so did the peak muscle torque about the knee (Figure 24). The maximum difference between torques was $0.3 \mathrm{Nm} / \mathrm{kg}$, and the maximum difference in contribution to the knee NJM was 20\% (Figure 25). While this seems like a substantial change, the vastus intermedius exhibited a maximum difference of $0.7 \mathrm{Nm} / \mathrm{kg}$ (Figure 24) and a maximum difference in contribution of $70 \%$ (Figure 25). Just like the rectus femoris torques followed the trend the muscle forces did, the vastus intermedius increases in peak force were matched by increases in muscle torque as load increased. The torque generated by the vastus intermedius was greater than the NJM for nearly the entire movement for all conditions (Figure 20), while the rectus femoris contribution wasn't even high enough to counteract the flexor torque created by the gastrocnemius and biceps femoris - long head until late in the movement.

\section{Figure 24}

\section{Relative Muscle Torques of the Individual Knee Extensors at the Knee}

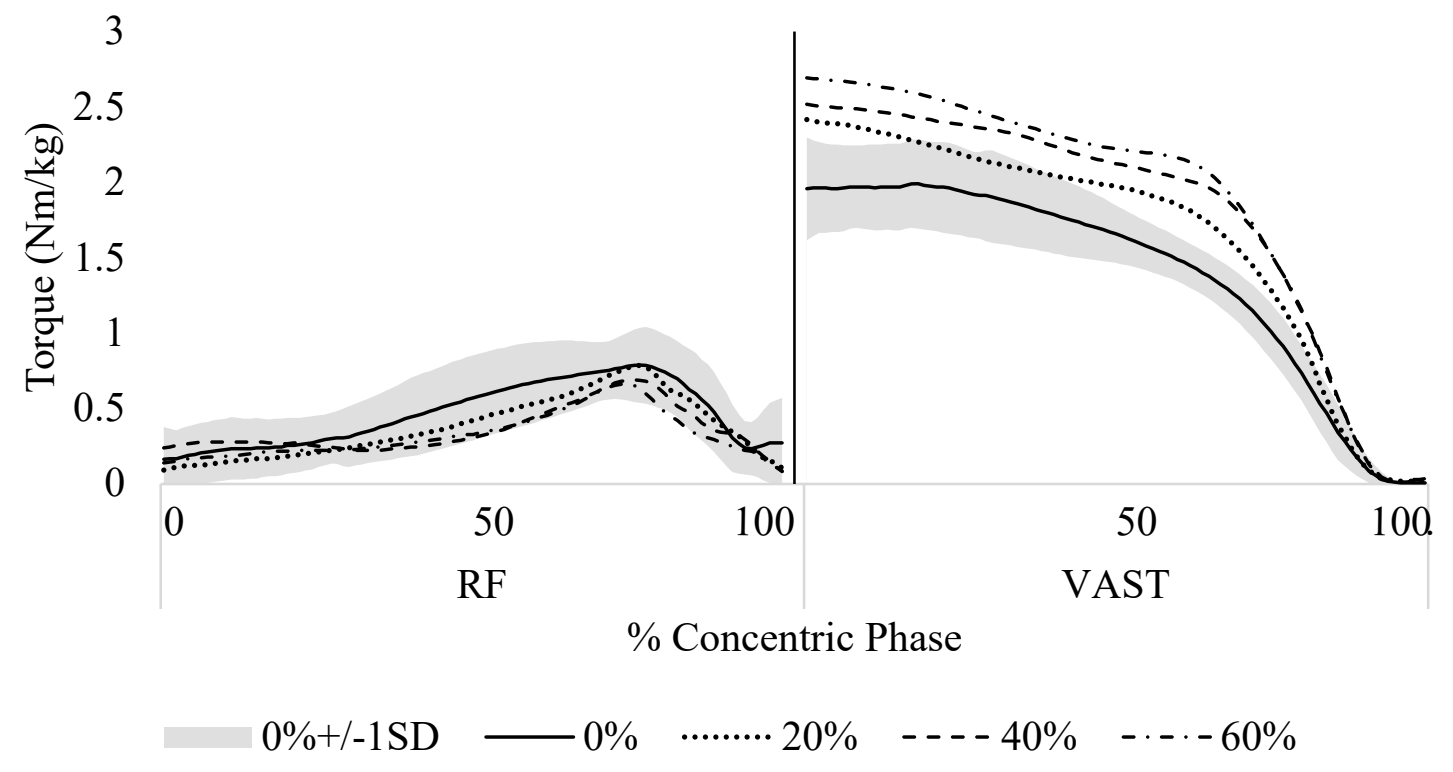




\section{Figure 25}

Contribution of the Individual Knee Extensors to the NJM at the Knee

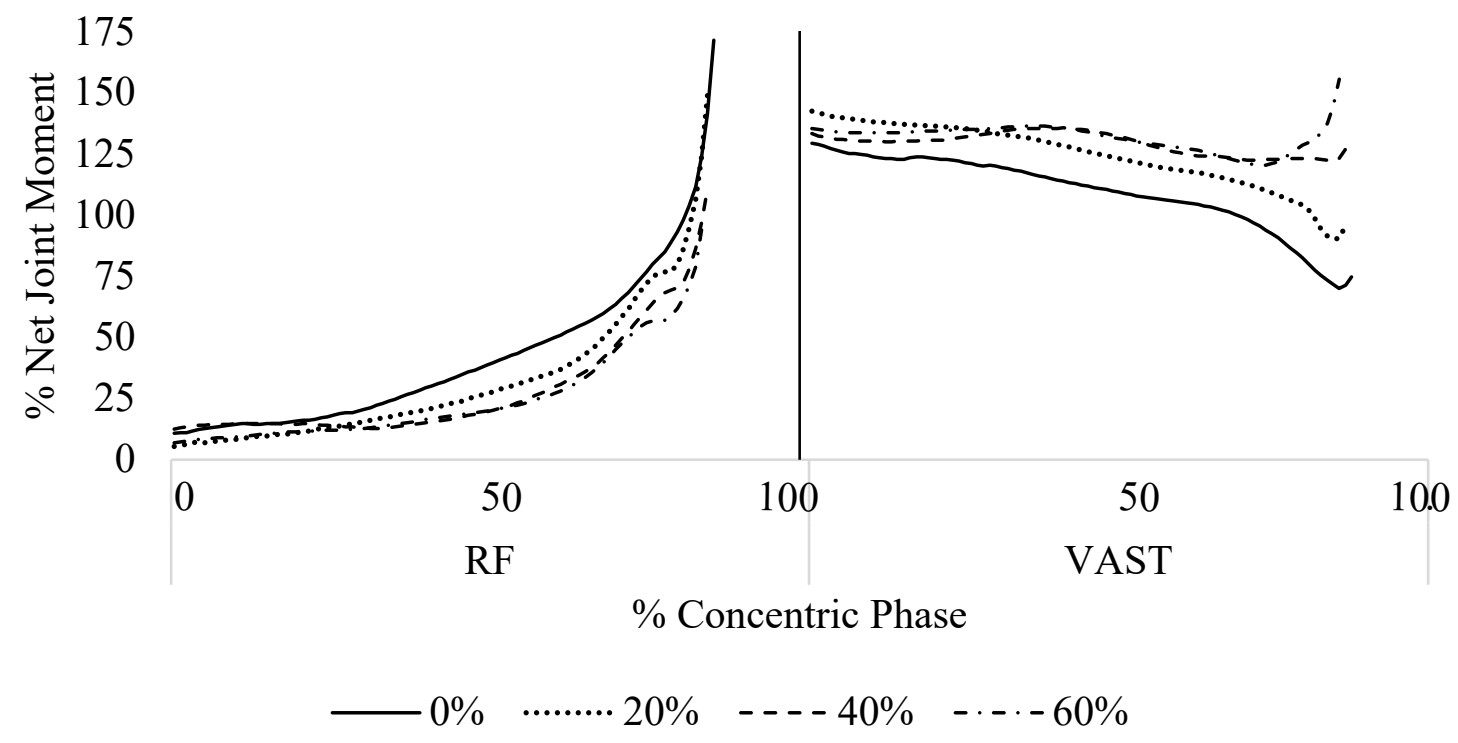

Note. Contribution was calculated as (individual muscle torque / NJM) * 100.

Ankle

Timeseries of the individual muscle torques and NJM at the ankle for each condition are presented in Figure 26, and the calculated contribution of each muscle to the NJM is presented in Figure 27. 


\section{Figure 26}

Relative Muscle Torques and Net Joint Moment at the Ankle During Each of the Conditions

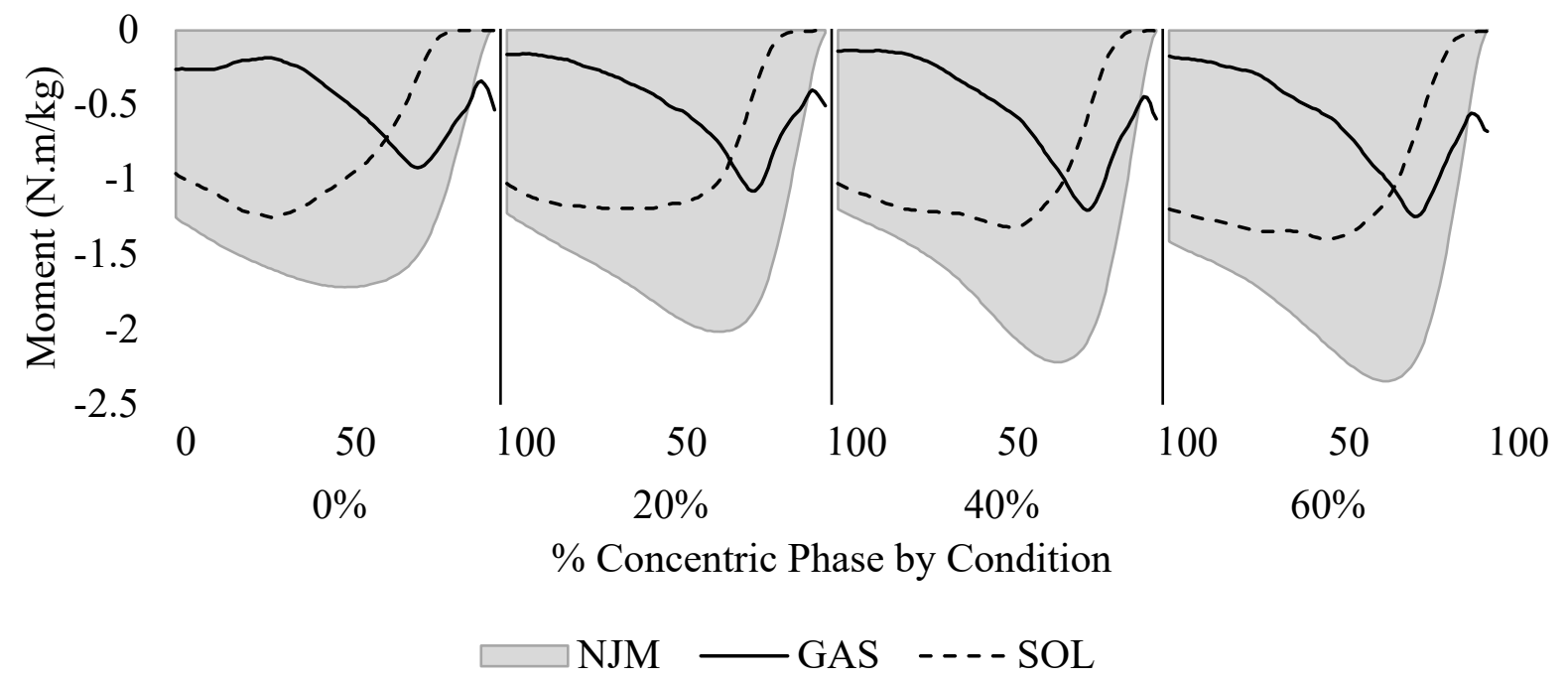

Note. NJM is shaded to designate net flexor (positive) versus net extensor (negative) patterns of the ankle joint moment and represents a sum of all individual muscle torques ( $\mathrm{n}=4$ muscles). Only the GAS and SOL are individually graphed due to being the largest muscle torques.

\section{Figure 27}

Contribution of Individual Muscles to the NJM at the Ankle

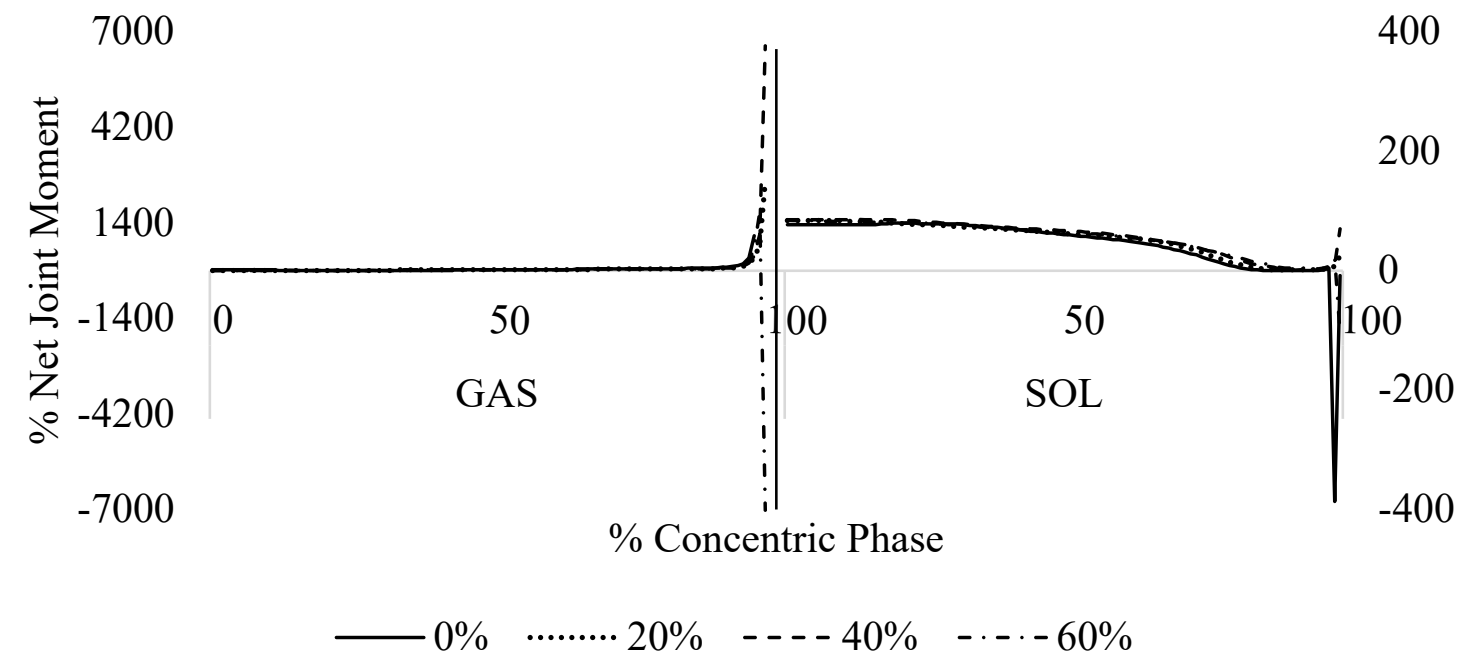

Note. Contribution was calculated as (individual muscle torque / NJM) * 100. 
Peak force of the soleus did not significantly differ across loads, and the peak muscle torque is visually similar across loads, although the timeseries show clear differences in torque magnitude at individual time points (Figure 28). Despite changes in the NJM, soleus contribution to the NJM remained similar across loading conditions (Figure 29). Similarly, the gastrocnemius displayed changes in peak muscle force and peak muscle torque across conditions (Figure 28), but contribution to the NJM did not substantially differ (Figure 29). Both plantarflexors exhibit increases in muscle torque with increases in external load, and the NJM also increased across conditions. Although there were clear differences in peak muscle torque for the gastrocnemius and no clear differences in peak muscle torque for the soleus, contribution remained consistent for both muscles across conditions. The soleus was responsible for the majority of the NJM until approximately $75 \%$ of the concentric phase, corresponding roughly to the time ankle angle begins to drastically change, at which time the gastrocnemius took over for the remainder of the concentric phase.

\section{Figure 28}

\section{Relative Muscle Torques of the Individual Plantarflexors at the Ankle}

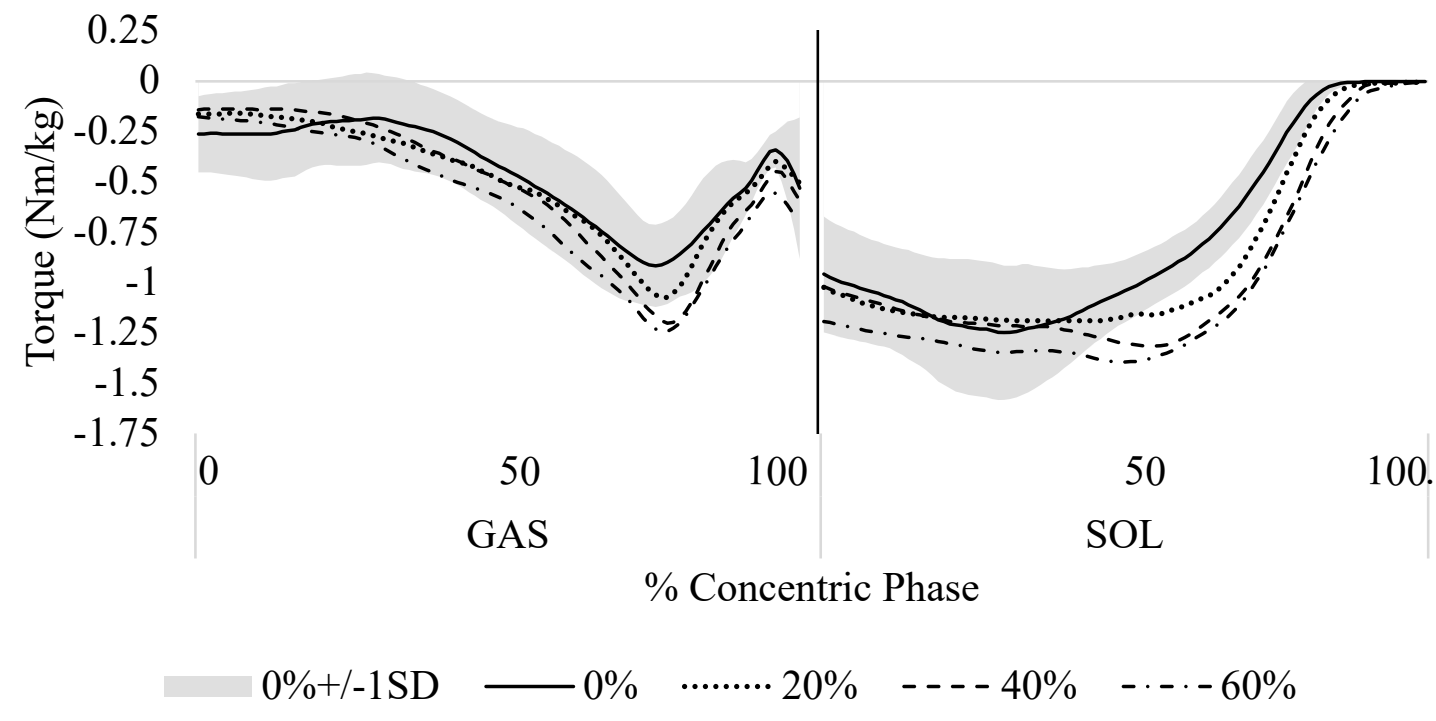




\section{Figure 29}

Contribution of Individual Plantarflexors to the NJM at the Ankle

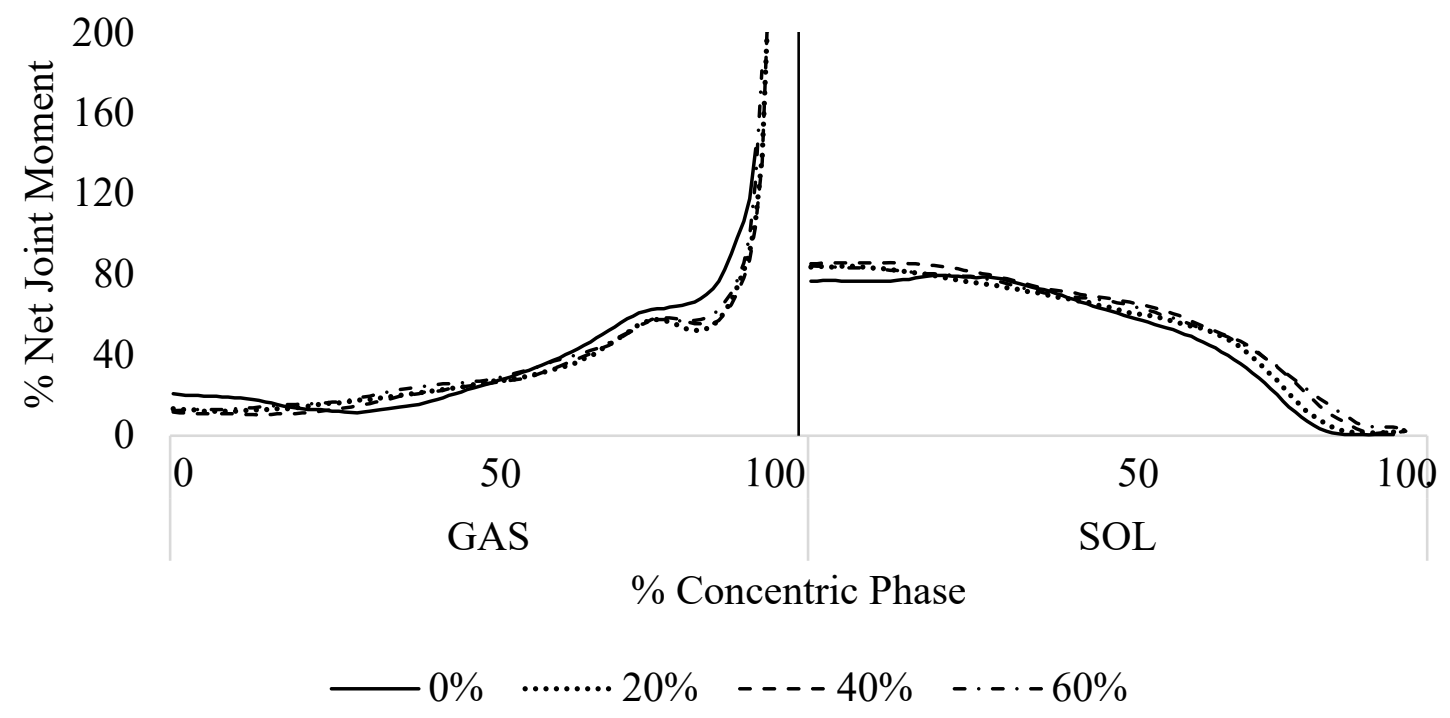

Note. Contribution was calculated as (individual muscle torque / NJM) * 100 .

\section{Discussion}

The purpose of this study was to describe individual muscle forces during the concentric phase of maximal unloaded vertical jumps and explore how those may change during the hexbar jump squat with increasing loads. Increases in peak muscle force were found for VAST and GAS with increasing load, and a decrease in peak muscle force was found for RF. No change in peak muscle force across loads was found for SOL, BFL, or GMAX.

Additionally, individual muscle torques and their contribution to the NJM were qualitatively analyzed. Muscle torque for the hip extensors was increased with increasing load, as well as the contribution to the NJM. Knee flexor torque was increased but contribution was unaltered by load, and rectus femoris torque and contribution decreased while vasti torque and contribution increased. Both plantarflexors exhibited increases in muscle torque with increasing loads but consistent contribution across conditions. 
The joint angles and joint moments calculated in OpenSim were qualitatively in good agreement with those determined by Visual 3D (C-Motion, Inc., Germantown, MD, USA). Additionally, angles obtained from OpenSim were within one standard deviation of those previously reported for unloaded countermovement jumps (Anderson \& Pandy, 1999; Fukashiro et al., 2005; Nagano et al., 2005). Joint moments obtained from OpenSim for the unloaded countermovement jump were also within one standard deviation of previously reported values (Aragón-Vargas \& Gross, 1997; Cleather et al., 2011, 2013; Vanrenterghem et al., 2004), and the trend of increasing moment with increasing external load corresponds to outcomes reported by Kipp et al. (2020b) and Moir et al. (2012).

Estimated muscle forces during an unloaded vertical jump have been reported previously (Bobbert et al., 1986; Cleather, 2019; Cleather et al., 2011; Cleather \& Cushion, 2019; Dahlkvist et al., 1982; Nagano et al., 2005; Pandy et al., 1990). Comparing the current values to a single study proved challenging due to the wide variation in muscle forces across studies; therefore, the minimum and maximum values of reported means were used to define a range for qualitative comparisons (Table 3). 


\section{Table 3}

Peak Muscle Forces as Compared to Literature

Notes: Absolute values are reported in $\mathrm{N}$, relative values are normalized to body weight (xBW).

\begin{tabular}{|c|c|c|c|c|c|c|}
\hline \multirow[b]{2}{*}{ Muscle } & \multicolumn{2}{|c|}{ Current Peak Forces } & \multicolumn{4}{|c|}{ Published Range } \\
\hline & Relative & Absolute & $\begin{array}{c}\text { Relative } \\
\text { Min. }\end{array}$ & $\begin{array}{c}\text { Relative } \\
\text { Max. }\end{array}$ & $\begin{array}{c}\text { Absolute } \\
\text { Min. }\end{array}$ & $\begin{array}{c}\text { Absolute } \\
\text { Max. }\end{array}$ \\
\hline RF & 2.5 & 2671 & $0.20^{\mathrm{a}}$ & $1.67^{\mathbf{b}}$ & & \\
\hline VAST & 7.89 & 8353 & $1.10^{\mathrm{c}}$ & $2.52^{\mathbf{b}}$ & & \\
\hline Quads. Total & 8.03 & & $6.05^{f}$ & $13.98^{\mathbf{b}}$ & $4000^{d}$ & $4315^{f}$ \\
\hline $\mathrm{BFL}$ & 3.71 & 3889 & $0.66^{\mathrm{c}}$ & $2.18^{b}$ & & \\
\hline Hams. Total & 5.03 & & $0.60^{a}$ & $5.17^{\mathbf{b}}$ & & \\
\hline GMAX & 2.53 & 2719 & $2.28^{c}$ & $4.63^{b}$ & $2200^{d}$ & $2200^{d}$ \\
\hline GAS & 2.14 & 2262 & $0.43^{\mathrm{c}}$ & $1.69^{b}$ & $708^{\mathbf{f}}$ & $3000^{e}$ \\
\hline SOL & 3.89 & 4138 & $4.37^{\mathrm{c}}$ & $8.2^{\mathbf{b}}$ & $2000^{d}$ & $3000^{e}$ \\
\hline PF Total & 3.85 & & $4.80^{c}$ & $9.89^{b}$ & $3000^{d}$ & $6000^{e}$ \\
\hline
\end{tabular}

Indicators are used to denote a reference for the reported value.

a (Cleather et al., 2011)

b (Cleather, 2019)

c (Cleather \& Cushion, 2019)

d (Pandy et al., 1990)

e (Bobbert et al., 1986)

f (Dahlkvist et al., 1982)

Differences in estimated muscle forces can be a product of differences, either singularly or cumulatively, in optimization techniques (Mokhtarzadeh et al., 2014; Roelker et al., 2020), the complexity, arrangement and architecture of the muscle models employed (Roelker et al., 2017), and/or the degrees of freedom assigned at each of the joints of the model (Mokhtarzadeh et al., 2014). Differences in estimated muscle forces can also be a propagation of uncertainties due to errors in marker placement, movement artifact, or variability of segment or muscle parameters 
(Myers et al., 2015). Assumptions made in some of the referenced studies may also contribute to differences in results. For example, Bobbert et al. (1986) assumed the same fiber optimal length and fiber composition between the gastrocnemius and soleus, which would affect the forcevelocity and force-length curves and likely overestimate output of the soleus. Similarly, Dahlkvist et al. (1982) assumed the opposition of external moments would be shared equally among all of the muscles responsible for a given joint motion, which would result in an altered distribution of forces when compared to optimization techniques such as static optimization.

Despite the current forces for individual muscles being outside the published ranges, when extensors were summed to represent functional groups (ex. quadriceps), totals were within the published range. The current model used consists of 54 actuators representing 20 bilateral lower extremity muscles and three bilateral trunk muscles. The quadriceps were represented by only the rectus femoris and the vastus intermedius, and the hamstrings were represented by only the biceps femoris - long head and biceps femoris - short head. The simplification of these two major muscle groups may explain why peaks forces for the entire muscle group were within published values but peaks of individual muscle forces were not.

\section{Muscle Forces}

Uniarticular muscles did not exhibit a consistent increase in peak force with increasing load, as increasing forces were only noted in VAST. Similarly, biarticular muscles, as a group, did not remain consistent across conditions. Only BFL was consistent across load; RF decreased with increasing load, and GAS increased.

When investigating muscle forces during the squat with increasing external loads, Kipp et al. (2020a) found increases in force in the gluteus maximus and the vasti (vastus medialis, vastus intermedius, vastus lateralis), as well as an inconsistent decrease in the rectus femoris. The 
authors noted no differences for forces of the gastrocnemius, soleus, or biceps femoris (Kipp et al., 2020a). The increase in the vasti force and the lack of change in biceps femoris force correspond with the results of the current study. The lack of change in soleus force is also consistent, but the difference in range of motion at the ankle, and therefore function of the plantarflexors, during squatting as compared to jumping is worth noting. An additional consideration is Kipp et al. (2020a) controlled speed of the movement, while the current study instituted a maximal speed and jump height effort. Muscle forces at lighter loads may have been reduced due a higher contraction velocity (Hill, 1938) if subjects were instead instructed to complete the motion as quickly as possible, not unlike a maximal effort jump.

Kipp and Kim (2020) found that during maximal effort vertical jumps, the gastrocnemius and soleus, as well as the vasti, contribute the most to acceleration of the whole body center of mass. The soleus, vasti and rectus femoris muscles were found to be operating close to maximal capacity, while the gastrocnemius and gluteus maximus were operating below their maximal capacities (Kipp \& Kim, 2020). The large reserve in force capability may explain the increases in GAS force seen with increasing load in the current study. Similarly, the smaller reserve for the vasti potentially explains why statistical increases were only noted between the heaviest and lightest loads, in comparison to the differences seen across all loads in GAS. Despite the gluteus maximus operating well below maximal capacity, the low potential to contribute to center of mass acceleration (Kipp \& Kim, 2020) may explain why there was no increase in GMAX force output with increasing external loads in the current results. Although the effect of load on the individual muscles examined is corroborated by other researchers and the changes noted logically make sense with other's findings, the effects cannot be explained strictly by examining the muscles based on whether they are uniarticular or biarticular muscles. 
When the current peak force results are grouped by joint rather than examined individually, it can be noted the hip extensors showed no change, the knee extensors showed changes in both muscles, and the plantarflexors showed a change in one muscle. Feeney et al. (2016) found a load-associated increase in knee work and power during jumps loaded with a weighted vest, while no significant trend was observed in the relative contributions of the hip or ankle. This trend is similar to what is seen in the current muscle forces at each of the joints, namely the knee extensors were most affected by external load. Similarly, individual joint contributions to the overall support moment change during squatting as external load increases (Flanagan \& Salem, 2008). Demands were not increased equally across joints as load increased, as the ankle and hip contributions to support moment increased while knee contribution decreased (Flanagan \& Salem, 2008). Increased demands during the current study were also not shared equally across joints, as measured by muscle forces, although the pattern of redistribution was different. The lack of increased demand at the hip observed in the current study can be corroborated by the minimal differences seen in peak hip NJM with increasing external loads.

A decrease in trunk incline, leading to a reduction in peak hip moment and power, has been shown to lead to an increase in knee joint power (Vanrenterghem et al., 2008), and increasing knee extensor strength has been shown to increase jump height through simulation (Bobbert \& Van Soest, 1994; Nagano \& Gerritsen, 2001). Hip abductors, adductors, and external rotators, although highly active during jumping, contribute little to jumping performance outside of stabilization (Nagano et al., 2005), and the gluteus maximus contributes little to center of mass acceleration (Kipp \& Kim, 2020). If the hip musculature stands to contribute little to jump performance, it seems reasonable that the body will look to increase output from the knee and 
ankle musculature as demands increase, but this is strictly speculation based on the trends found in the current data.

\section{Muscle Torques}

Net joint moments are often used as an indication of the neuromechanical demands during a given task (Flanagan \& Salem, 2008; Kipp et al., 2020b), and represent a measure of the net external + internal torques muscles must overcome to create movement about a joint. Thus, the contribution of individual muscles to the NJM indicates how the mechanical demands of a task are met (Kipp et al., 2020b), and potentially how changes in muscle force are translated to changes in mechanical output.

The NJMs in the current study are similar in magnitude and pattern to those previously reported (Aragón-Vargas \& Gross, 1997; Cleather et al., 2011, 2013; Vanrenterghem et al., 2004), and the trend of increasing moment with increasing external load corresponds to previous literature (Kipp et al., 2020b; Moir et al., 2012). Additionally, the sum of individual muscle torques was equivalent to the NJM determined by ID, indicating muscle torque values are reasonable and the method of handling biarticular muscles was valid. The NJM at all three joints increased as load increased; however, the ankle showed much more pronounced differences than the other two joints.

Similar to the NJM, both hip extensors showed an increase in torque with the addition of external load, but not substantial differences between loads. The gluteus maximus values were roughly half of the biceps femoris - long head values and the contribution of each muscle to the NJM was similarly scaled. The rectus femoris creates a flexion torque about the hip, and exhibited decreasing values with increasing load; furthermore, the increasing magnitude in the NJM resulted in a substantial decrease in negative contribution from the rectus femoris as load 
increased. The substantial decrease in negative contribution from the rectus femoris, in combination with the increased extensor torques created by the hip extensors, is responsible for the increases in the NJM as load increased. Although changes in torque were noted for the hip extensors during portions of the concentric phase, no changes in peak force were found for these muscles. Differences were noted in hip angle with the addition of external load, and as such the moment arm of the muscles was altered, explaining why changes in muscle torque were not matched by changes in muscle force.

The gastrocnemius torque at the knee had a maximum difference of $31 \%$ between loads, and the biceps femoris - long head had slightly larger differences in muscle torques at the beginning of the movement (37\%); however, the summed flexor moment varied by a maximum of $32 \%$ between conditions and contribution to the NJM was consistent across conditions. Although the knee flexors created a negative contribution to the NJM, the quadriceps created a considerably larger torque in order to extend the knee. Peak rectus femoris torque decreased with increasing load, although changes between conditions were small in comparison to the vastus intermedius. Torque generated by the vastus intermedius increased with load and was greater than the NJM for all conditions. Although there was an alteration in distribution between muscles such that the vastus contribution increased with load, changes in the NJM about the knee were primarily driven by changes in the vastus torque. The changes to torque of the individual quadriceps and the contributions to the NJM correspond to the findings in the peak muscle forces. Increasing external load created an increased NJM about the knee and prompted a shift in force production from the rectus femoris to the vastus intermedius.

Peak torque of the gastrocnemius at the ankle was increased with increasing loads, while the soleus peak torque was similar across conditions; however, if the soleus data is examined at 
individual time points, increases can be noted. Despite increases in torque from both muscles, due to changes in the NJM, contributions remained similar across loading conditions. The change in NJM cannot be explained by just investigating peak muscle torques across conditions, and instead should be examined at the level of the timeseries. Despite a difference in changes to torque values between the plantarflexors, contribution from both muscles remains consistent across loads. Interestingly, peak force values only significantly increased for the gastrocnemius. Similar to changes in peak torque values not fully explaining changes in the NJM, changes in peak force do not give the full picture either. While peak soleus force did not change across loads, clear differences can be seen at individual timepoints. Increasing load did not lead to increases in peak muscle force or torque during the concentric phase for the soleus, but changes in timing of peak values indicates load does play a factor in muscle function at the ankle.

Previous literature on muscle torques during jumping has found the torque created by the vasti to be greater than the NJM, similar to the current results, due to the need to overcome the antagonistic action of the gastrocnemius at the knee (van Soest et al., 1993). Previous research has also included investigating muscle contributions during jumping and found the dominant contributors to trunk segment acceleration were the gastrocnemius, the vasti, the gluteus maximus, and the soleus; the hamstrings and rectus femoris were found to contribute little (Pandy \& Zajac, 1991). While the current results for the torque contributions from the quadriceps and plantarflexors correspond to these findings, the results for the hip extensors differ. Instead of substantial contributions from the gluteus maximus and minimal contribution of the hamstrings to NJM, the opposite was found: the hamstrings created twice the contribution of the gluteus maximus. As discussed above, differences could be a result of differences in measurement or calculation methods, a difference in the model used, or different muscle parameters. 
Squatting, while not at the same speed, is a similar motion to jump squats, and can also involve the application of different loads. With an increase in external load, Kipp et al. (2020b) reported increases in the NJM at the hip, knee, and ankle. At the hip, both the gluteus maximus and hamstring muscle torques increased with additional loads, and the ratio between these torques did not change across conditions (Kipp et al., 2020b). Results of the current study correspond with these findings, as well as the finding that the hamstrings contribute more to the hip extension NJM than the gluteus maximus (Kipp et al., 2020b). At the knee, in line with the current findings, Kipp et al. (2020b) reported an increase in the vasti and hamstring torques, as well as a slight decrease in torque from the rectus femoris as external load increased. Additionally, although the ankle range of motion is different between a squat and a vertical jump, the results are worth noting. An increase in torque created by the soleus with increasing load corresponds to the current results, but Kipp et al. (2020b) found no significant changes in gastrocnemius torque during the squat. This was not the case for the current study, but the difference in findings may be due to the difference in range of motion. The gastrocnemius did not become the majority contributor to the NJM until roughly the same time in the concentric phase as the ankle angle begins to change, indicating the difference in range of motion at the ankle is of particular importance when comparing squats to jumping.

The gastrocnemius is a biarticular muscle and as such, knee range of motion is also important to consider when investigating muscle function. Kipp et al. (2020b) speculated the position of deep knee flexion experienced during a squat negatively impacted the force-length relationship for the gastrocnemius to such a degree that the potential to generate a plantarflexion torque was limited. The lack of deep knee flexion and a comparatively more extended position during jumping may explain the greater differences found in gastrocnemius torque in the current 
study. The effect of the biarticularity of the gastrocnemius is largely dependent on the relationship of the moment arm at the knee as a function of knee angle (van Soest et al., 1993). While some research suggests the importance of the gastrocnemius has nothing to do with the biarticularity of the muscle (Pandy \& Zajac, 1991), others researchers argue the moment arm of the gastrocnemius at the knee increases with knee extension (van Soest et al., 1993) and thus the gastrocnemius plays an important role because it is a biarticular muscle. During the current investigation, moment arm of the gastrocnemius increased as the knee joint extended, indicating knee joint angle may have played an important role in gastrocnemius force and torque. Because the knee is extending, the velocity of shorting of the biarticular gastrocnemius is reduced (van Soest et al., 1993). The dynamic coupling of the knee and ankle joint during jumping further perpetuates this effect, because as the knee extends, the ankle is also extending (van Ingen Schenau et al., 1990), slowing velocity of shortening even more. The difference in shortening velocity, as a product of a different range of motion at both the knee and ankle, may explain in part why more significant changes were found during the current investigation as compared to that of squats (Kipp et al., 2020b). The slower contraction velocity allows the gastrocnemius to operate in a more favorable portion of the force-velocity curve (Hill, 1938; van Soest et al., 1993), potentially explaining why peak gastrocnemius forces continued to increase with increasing load.

Just like was found during vertical jumping, the vasti tend to overcontribute to the knee NJM during a sidestep motion (Maniar et al., 2019). The soleus is known to contribute to knee stability during the single leg stance phase of gait by controlling tibial internal rotation and if contribution of the soleus is reduced, an increase in vasti contribution is needed to prevent knee collapse (Neptune et al., 2001). Because the soleus has a large proportion of slow twitch muscle 
fibers, force production is particularly sensitive to velocity of shortening (Hamner \& Delp, 2013). Given that the hexbar squat jump is a movement that is performed quickly, a reduction in force capability of the soleus may also contribute to the increased contribution needed from the vasti to successfully complete the motion. During the current investigation, peak soleus force did not significantly differ across loads. Additionally, peak gastrocnemius force increased across loads. The lack of increase in soleus force to contribute to knee stability, in combination with the increased gastrocnemius force creating antagonistic action at the knee joint, contributes to why peak force of the vastus intermedius was increased with increasing load.

How individual muscles contribute to the NJM is an indication of how mechanical demands of the task are met (Kipp et al., 2020b) and knowledge of how the NJM is distributed across different muscles is important to understand when loading a movement, particularly if the distribution is altered by the addition of load. The results of this study indicate the addition of external load will affect the muscular demands differently at each of the lower extremity joints.

At the ankle, contribution from the soleus and gastrocnemius remained relatively consistent across loads, indicating hexbar jump squats create a similar mechanical demand as unloaded jumps and therefore are a potential method of training the ankle for jumping at a higher intensity than can be achieved with body weight alone. At the knee and hip, contributions were altered. At the hip, the antagonistic contribution of the rectus femoris was reduced with increasing load, while contribution from both hip extensors was increased during the early portion of the concentric phase. Loaded jumps place a different mechanical demand on the hip joint, which is an important consideration if improved vertical jump performance is desired. At the knee, antagonistic contribution of the gastrocnemius and biceps femoris - long head was not consistently altered by increases in external load; however, the quadriceps were affected by 
increasing loads. The contribution from the rectus femoris decreased while the contribution from the vasti consequently increased, indicating loaded jumps can be used to elicit greater vasti output than body weight alone; however, the altered demands may reduce transferability of increased strength gains (Bobbert \& Van Soest, 1994).

\section{Limitations}

The limitations are recognized. The location of the hexbar during the jumps was not tracked during data collection. This meant the location of the external load could not be accurately modeled as a separate entity. To account for the mass of the external load, it was instead applied to the entire subject. The hexbar places the mass of the load in close proximity to the center of mass (Swinton et al., 2012); therefore, if the hexbar is thought to load the center of mass, by distributing the mass of the hexbar throughout the entire body the ultimate location of the combined center of mass of the system will be relatively unchanged. The inertial properties of individual segments would be affected; however, there was close agreement between the moments calculated by OpenSim with the added mass and those calculated by Visual3D with no added mass. The distribution of the hexbar mass across the entire body did not create large discrepancies in lower extremity kinetics, and thus indirectly supports the current method.

Another limitation is the difference in the strength characteristics of the current subjects as compared to those used to set the muscle parameters of the model. The model uses parameters specifically set to best represent older adults 65-86 years old (Thelen, 2003). Both isometric strength and maximum contraction velocity were reduced from the values used for young adults (Thelen, 2003). Although isometric strength values were increased to account for the athletic status of the current subjects, isokinetic strength testing was not performed, so the adjusted muscle parameters are simply best estimates based on previous literature (Cleather et al., 2011; 
Tomescu et al., 2018). Given the maximal effort required during what is generally considered a powerful movement, these differences are important to note.

While not specifically a limitation, the method used to calculate and analyze contribution data deserves a more thorough discussion. Contribution to the NJM was handled in two unique ways; contribution was calculated at the level of the group, and contribution curves were truncated to only represent contribution to the extensor portion of the NJM. The NJM for all three lower extremity joints crosses zero or comes very near to zero. The reason for this is what is deemed the anatomical constraint (van Ingen Schenau et al., 1990). In order to preserve integrity of the rapidly extending joint, the antagonistic muscles, and the resulting flexor torque, must be used to reduce velocity of the joint to prevent hyperextension (van Ingen Schenau et al., 1990). Because the NJM is the denominator in the equation, dividing by the very small NJM values immediately prior to and following the zero point leads to extremely large magnitudes for the resulting contribution values, as well as a near vertical line connecting these points at the instant the NJM crosses zero. The variation in timing of these zero points between subjects, and even between trials, led to unrepresentative curves when values were averaged. Contribution curves were instead calculated using the group averages for muscle torques and NJMs in order to have a curve that was representative of the true average and was not skewed by the variation in timing of extreme values. Although the calculated contribution values surrounding the zero point are mathematically valid, for comparison to the rest of the concentric phase they were deemed physiologically unreasonable. The contribution timeseries were thus truncated to explore just the portion of the concentric phase when the NJM was an extensor moment. The final 3 data points of this extensor portion were also removed in order to mitigate any effect of the small divisor and instead include only physiologically relevant data points. 


\section{Conclusion}

Individual muscle forces were estimated during maximal effort vertical jumps and hexbar jumps under loads up to $60 \% 1 \mathrm{RM}$. Additionally, individual muscle torques and the contribution to the NJM were investigated. The gastrocnemius increased peak force but contribution from individual plantarflexors remained consistent across conditions, indicating loaded jumps increase the intensity of jumping without altering mechanical demands; however, changes in timing of peak muscle force and torque values indicates load does play a factor in muscle function at the ankle and should be considered. The vasti increased peak force while rectus femoris concomitantly decreased peak force. Torque contribution demands accordingly shifted further onto the vasti with increasing load, indicating loaded jumps can be used to elicit greater output from uniarticular knee extensors. Peak force of the hip extensors was unaffected by increases in external load, but a decrease in hip flexor torque led to higher contribution from the hip extensors than is seen during unloaded jumping; therefore, loaded jumping place more emphasis on hip extensors than unloaded vertical jumps.

Knowledge of how mechanical demand is distributed across individual muscles is important to understand when loading a movement, particularly if the distribution is altered by the addition of load. The results of this study indicate loaded hexbar jumping is not simply a higher intensity version of vertical jumping, and the lower extremity joints and corresponding musculature are not impacted equally by the addition of load. The varied effect of load on mechanical demands at the lower extremity joints, and thus force and torque output from individual muscles, is important to consider when using loaded jumps as part of training for athletic performance. 


\section{REFERENCES}

Anderson, F. C., \& Pandy, M. G. (1999). A dynamic optimization solution for vertical jumping in three dimensions. Computer Methods in Biomechanics and Biomedical Engineering, 2(3), 201-231. https://doi.org/10.1080/10255849908907988

Aragón-Vargas, L. F., \& Gross, M. M. (1997). Kinesiological factors in vertical jump performance: Differences among individuals. Journal of Applied Biomechanics, 13(1), 24-44. https://doi.org/10.1123/jab.13.1.24

Bobbert, M. F., Huijing, P. A., \& van Ingen Schenau, G. J. (1986). An estimation of power output and work done by the human triceps surae musle-tendon complex in jumping. Journal of Biomechanics, 19(11), 899-906. https://doi.org/10.1016/0021-9290(86)901855

Bobbert, M. F., \& Van Soest, A. J. (1994). Effects of muscle strengthening on vertical jump height: A simulation study. Medicine \& Science in Sports \& Exercise, 26(8), 1012-1020.

Cleather, D. (2019). Neural network based approximation of muscle and joint contact forces during jumping and landing. Journal of Human Performance and Health, 1(1). https://doi.org/10.31236/osf.io/f7cg3

Cleather, D., \& Cushion, E. (2019). Muscular coordination during vertical jumping. Journal of Human Performance and Health, 1(1). https://doi.org/10.31236/osf.io/68mnd

Cleather, D., Goodwin, J. E., \& Bull, A. M. J. (2011). An optimization approach to inverse dynamics provides insight as to the function of the biarticular muscles during vertical jumping. Annals of Biomedical Engineering, 39(1), 147-160.

https://doi.org/10.1007/s10439-010-0161-9 
Cleather, D., Goodwin, J. E., \& Bull, A. M. J. (2013). Intersegmental moment analysis characterizes the partial correspondence of jumping and jerking. Journal of Strength and Conditioning Research, 27(1), 89-100. https://doi.org/10.1519/JSC.0b013e31825037ee

Dahlkvist, N. J., Mayo, P., \& Seedhom, B. B. (1982). Forces during squatting and rising from a deep squat. Engineering in Medicine, 11(2), 69-76. https://doi.org/10.1243/EMED_JOUR_1982_011_019_02

Delp, S. L., Anderson, F. C., Arnold, A. S., Loan, P., Habib, A., John, C. T., Guendelman, E., \& Thelen, D. G. (2007). OpenSim: Open-source software to create and analyze dynamic simulations of movement. IEEE Transactions on Biomedical Engineering, 54(11), 19401950. https://doi.org/10.1109/TBME.2007.901024

Epley, B. (1985). Poundage Chart. In Boyd Epley Workout. Body Enterprises.

Feeney, D., Stanhope, S. J., Kaminski, T. W., Machi, A., \& Jaric, S. (2016). Loaded vertical jumping: Force-velocity relationship, work, and power. Journal of Applied Biomechanics, 32(2), 120-127. https://doi.org/10.1123/jab.2015-0136

Flanagan, S. P., \& Salem, G. J. (2008). Lower extremity joint kinetic responses to external resistance variations. Journal of Applied Biomechanics, 24(1), 58-68. https://doi.org/10.1123/jab.24.1.58

Fukashiro, S., Besier, T. F., Barrett, R., Cochrane, J., Nagano, A., \& Lloyd, D. G. (2005). Direction control in standing horizontal and vertical jumps. International Journal of Sport and Health Science, 3(Special_Issue_2), 272-279. https://doi.org/10.5432/ijshs.3.272 
Gregoire, L., Veeger, H., Huijing, P., \& van Ingen Schenau, G. (1984). Role of mono- and biarticular muscles in explosive movements. International Journal of Sports Medicine, 05(06), 301-305. https://doi.org/10.1055/s-2008-1025921

Hamner, S. R., \& Delp, S. L. (2013). Muscle contributions to fore-aft and vertical body mass center accelerations over a range of running speeds. Journal of Biomechanics, 46(4), 780-787. https://doi.org/10.1016/j.jbiomech.2012.11.024

Hicks, J. L. (2018). Simulation with OpenSim—Best Practices. https://simtkconfluence.stanford.edu. https://simtkconfluence.stanford.edu:8443/display/OpenSim33/Simulation+with+OpenSim++ Best + Practices?showChildren $=$ true

Hill, A. V. (1938). The heat of shortening and the dynamic constants of muscle. Proceedings of the Royal Society of London. Series B - Biological Sciences, 126(843), 136-195. https://doi.org/10.1098/rspb.1938.0050

Jacobs, R., \& Bobbert, M. F. (1996). Mechanical output from individual muscles during explosive leg extensions: The role of biarticular muscles. Journal of Biomechanics, 29(4), $513-523$.

Kellis, E., Arambatzi, F., \& Papadopoulos, C. (2005). Effects of load on ground reaction force and lower limb kinematics during concentric squats. Journal of Sports Sciences, 23(10), 1045-1055. https://doi.org/10.1080/02640410400022094

Kipp, K., \& Kim, H. (2020). Relative contributions and capacities of lower extremity muscles to accelerate the body's center of mass during countermovement jumps. Computer Methods in Biomechanics and Biomedical Engineering, 1-8. https://doi.org/10.1080/10255842.2020.1772764 
Kipp, K., Kim, H., \& Wolf, W. I. (2020a). Muscle forces during the squat, split squat, and stepup across a range of external loads in college-aged men. Journal of Strength and Conditioning Research, Publish Ahead of Print. https://doi.org/10.1519/JSC.0000000000003688

Kipp, K., Kim, H., \& Wolf, W. I. (2020b). Muscle-specific contributions to lower extremity net joint moments while squatting with different external loads. Journal of Strength and Conditioning Research, Publish Ahead of Print. https://doi.org/10.1519/JSC.0000000000003874

Lees, A., Vanrenterghem, J., \& De Clercq, D. (2004). The maximal and submaximal vertical jump: Implications for strength and conditioning. The Journal of Strength and Conditioning Research, 18(4), 787. https://doi.org/10.1519/14093.1

Lockie, R. G., \& Lazar, A. (2017). Exercise technique: Applying the hexagonal bar to strength and power training. Strength and Conditioning Journal, 39(5), 24-32. https://doi.org/10.1519/SSC.0000000000000327

Lu, T. W., \& O’Connor, J. J. (1999). Bone position estimation from skin marker co-ordinates using global optimisation with joint constraints. Journal of Biomechanics, 32(2), 129134. https://doi.org/10.1016/S0021-9290(98)00158-4

Maeda, G. A. (2018). Joint Contributions of Loading Variations for the Hexagonal Barbell Countermovement Jump. Illinois State University.

Maniar, N., Schache, A. G., Cole, M. H., \& Opar, D. A. (2019). Lower-limb muscle function during sidestep cutting. Journal of Biomechanics, 82, 186-192. https://doi.org/10.1016/j.jbiomech.2018.10.021 
Mcbride, J. M., Triplett-Mcbride, T., Davie, A., \& Newton, R. U. (2002). The effect of heavyvs. Light-load jump squats on the development of strength, power, and speed. Journal of Strength and Conditioning Research, 16(2), 75-82.

Moir, G. L., Gollie, J. M., Davis, S. E., Guers, J. J., \& Witmer, C. A. (2012). The effects of load on system and lower-body joint kinetics during jump squats. Sports Biomechanics, 11(4), 492-506. https://doi.org/10.1080/14763141.2012.725426

Mokhtarzadeh, H., Perraton, L., Fok, L., Muñoz, M. A., Clark, R., Pivonka, P., \& Bryant, A. L. (2014). A comparison of optimisation methods and knee joint degrees of freedom on muscle force predictions during single-leg hop landings. Journal of Biomechanics, 47(12), 2863-2868. https://doi.org/10.1016/j.jbiomech.2014.07.027

Myers, C. A., Laz, P. J., Shelburne, K. B., \& Davidson, B. S. (2015). A Probabilistic approach to quantify the impact of uncertainty propagation in musculoskeletal simulations. Annals of Biomedical Engineering, 43(5), 1098-1111. https://doi.org/10.1007/s10439-014-1181-7

Nagano, A., \& Gerritsen, K. G. M. (2001). Effects of Neuromuscular Strength Training on Vertical Jumping Performance-A Computer Simulation Study. Journal of Applied Biomechanics, 17(2), 113-128. https://doi.org/10.1123/jab.17.2.113

Nagano, A., Komura, T., Fukashiro, S., \& Himeno, R. (2005). Force, work and power output of lower limb muscles during human maximal-effort countermovement jumping. Journal of Electromyography and Kinesiology, 15(4), 367-376. https://doi.org/10.1016/j.jelekin.2004.12.006

Neptune, R. R., Kautz, S. A., \& Zajac, F. E. (2001). Contributions of the individual ankle plantar flexors to support, forward progression and swing initiation during walking. Journal of Biomechanics, 34(11), 1387-1398. https://doi.org/10.1016/S0021-9290(01)00105-1 
Pandy, M. G., \& Zajac, F. E. (1991). Optimal muscular coordination strategies for jumping. Journal of Biomechanics, 24(1), 1-10. https://doi.org/10.1016/0021-9290(91)90321-D

Pandy, M. G., Zajac, F. E., Sim, E., \& Levine, W. S. (1990). An optimal control model for maximum-height human jumping. Journal of Biomechanics, 23(12), 1185-1198. https://doi.org/10.1016/0021-9290(90)90376-E

Prilutsky, B. I., \& Zatsiorsky, V. M. (1994). Tendon action of two-joint muscles: Transfer of mechanical energy between joints during jumping, landing, and running. Journal of Biomechanics, 27(1), 25-34. https://doi.org/10.1016/0021-9290(94)90029-9

Robertson, D. G. E., Wilson, J.-M. J., \& Pierre, T. A. St. (2008). Lower extremity muscle functions during full squats. Journal of Applied Biomechanics, 24(4), 333-339. https://doi.org/10.1123/jab.24.4.333

Roelker, S. A., Caruthers, E. J., Baker, R. K., Pelz, N. C., Chaudhari, A. M. W., \& Siston, R. A. (2017). Interpreting musculoskeletal models and dynamic simulations: Causes and effects of differences between models. Annals of Biomedical Engineering, 45(11), 2635-2647. https://doi.org/10.1007/s10439-017-1894-5

Roelker, S. A., Caruthers, E. J., Hall, R. K., Pelz, N. C., Chaudhari, A. M. W., \& Siston, R. A. (2020). Effects of optimization technique on simulated muscle activations and forces. Journal of Applied Biomechanics, 36(4), 259-278. https://doi.org/10.1123/jab.2018-0332

Swinton, P. A., Stewart, A. D., Lloyd, R., Agouris, I., \& Keogh, J. W. L. (2012). Effect of load positioning on the kinematics and kinetics of weighted vertical jumps. Journal of Strength and Conditioning Research, 26(4), 906-913. https://doi.org/10.1519/JSC.0b013e31822e589e 
Thelen, D. G. (2003). Adjustment of muscle mechanics model parameters to simulate dynamic contractions in older adults. Journal of Biomechanical Engineering, 125(1), 70-77. https://doi.org/10.1115/1.1531112

Tomescu, S. S., Bakker, R., Beach, T. A. C., \& Chandrashekar, N. (2018). The effects of filter cutoff frequency on musculoskeletal simulations of high-impact movements. Journal of Applied Biomechanics, 34(4), 336-341. https://doi.org/10.1123/jab.2017-0145

Turner, A. P., Unholz, C. N., Potts, N., \& Coleman, S. G. S. (2012). Peak power, force, and velocity during jump squats in professional rugby players. Journal of Strength and Conditioning Research, 26(6), 1594-1600. https://doi.org/10.1519/JSC.0b013e318234ebe5

Turner, T. S., Tobin, D. P., \& Delahunt, E. (2015a). Peak power in the hexagonal barbell jump squat and its relationship to jump performance and acceleration in elite rugby union players. Journal of Strength and Conditioning Research, 29(5), 1234-1239. https://doi.org/10.1519/JSC.0000000000000770

Turner, T. S., Tobin, D. P., \& Delahunt, E. (2015b). Optimal loading range for the development of peak power output in the hexagonal barbell jump squat. Journal of Strength and Conditioning Research, 29(6), 1627-1632.

https://doi.org/10.1519/JSC.0000000000000802

van Ingen Schenau, G., Bobbert, M. F., \& Van Soest, A. J. (1990). The Unique Action of BiArticular Muscles in Leg Extensions. In Multiple Muscle Systems: Biomechanics and Movement Organization. Springer. 
van Soest, A. J., Schwab, A. L., Bobbert, M. F., \& van Ingen Schenau, G. J. (1993). The influence of the biarticularity of the gastrocnemius muscle on vertical-jumping achievement. Journal of Biomechanics, 26(1), 1-8. https://doi.org/10.1016/00219290(93)90608-H

Vanrenterghem, J., Lees, A., \& Clercq, D. D. (2008). Effect of forward trunk inclination on joint power output in vertical jumping. Journal of Strength and Conditioning Research, 22(3), 708-714. https://doi.org/10.1519/JSC.0b013e3181636c6c

Vanrenterghem, J., Lees, A., Lenoir, M., Aerts, P., \& De Clercq, D. (2004). Performing the vertical jump: Movement adaptations for submaximal jumping. Human Movement Science, 22(6), 713-727. https://doi.org/10.1016/j.humov.2003.11.001

Whittlesey, S. N., \& Robertson, D. G. E. (2014). Two-Dimensional Inverse Dynamics. In D. G. E. Robertson, G. E. Caldwell, J. Hamill, G. Kamen, \& S. N. Whittlesey, Research methods in biomechanics (Second edition). Human Kinetics.

Zajac, F. E. (1993). Muscle coordination of movement: A perspective. Journal of Biomechanics, 26, 109-124. https://doi.org/10.1016/0021-9290(93)90083-Q 


\section{APPENDIX A: MARKER PLACEMENT AND LOCATIONS}

Table A-1

Detailed Marker Placement

\begin{tabular}{|c|c|}
\hline Marker Name & Location \\
\hline Sacrum & Vertical midline of the sacrum \\
\hline L\&R IC & Iliac crest of pelvis \\
\hline L\&R Troch & Greater trochanter of the femur \\
\hline R Thigh Cluster & Placed on lateral aspect of thigh \\
\hline RThigh_1 & Anterior superior corner \\
\hline RThigh_2 & Anterior inferior corner \\
\hline RThigh_3 & Posterior inferior corner \\
\hline RThigh_4 & Posterior superior corner \\
\hline L Thigh Cluster & Placed on lateral aspect of thigh \\
\hline LThigh_1 & Posterior superior corner \\
\hline LThigh_2 & Posterior inferior corner \\
\hline LThigh_3 & Anterior inferior corner \\
\hline LThigh_4 & Posterior superior corner \\
\hline L\&R LKnee & Lateral epicondyle of femur \\
\hline L\&R MKnee & Medial epicondyle of femur \\
\hline R Shank Cluster & Placed on lateral aspect of shank \\
\hline RShank_1 & Anterior superior corner \\
\hline RShank_2 & Anterior inferior corner \\
\hline RShank_3 & Posterior inferior corner \\
\hline L Shank Cluster & Placed on lateral aspect of shank \\
\hline LShank_1 & Posterior inferior corner \\
\hline LShank_2 & Anterior inferior corner \\
\hline LShank_3 & Anterior superior corner \\
\hline L\&R Heel & Posterior aspect of calcaneus \\
\hline L\&R LAnkle & Lateral malleolus \\
\hline L\&R MAnkle & Medial malleolus \\
\hline L\&R MToe & Head of 1 st metatarsal \\
\hline L\&R LToe & Head of 5th metatarsal \\
\hline
\end{tabular}




\section{APPENDIX B: DETAILED WORKFLOW}

Vicon

- Marker data was digitized according to marker set, gaps were filled with pattern and rigid body fill

- "Lower Extremity - Dynamic" pipeline was run. GRF data was filtered at $300 \mathrm{~Hz}$ and trajectory data were filtered at $6 \mathrm{~Hz}$. Data was exported as a .csv.

\section{MATLAB}

- A custom MATLAB script was used to transform .csv file into trajectory (.trc) and GRF (.mot) files for OpenSim.

- Columns for the trajectory of calibration markers were removed from the .csv before processing, as well as rows containing any blank cells (indicating a gap).

\section{OpenSim - Scaling}

- The generic model was scaled using the static calibration trial data and length of segments was adjusted based on distance between markers.

- $\quad$ Torso was scaled to length of femur due to lack of upper extremity markers

- Pelvis was scaled using the LIC and RIC markers, and was scaled only in the X and Z directions.

- All other segments were scaled uniformly using lateral markers at either end of the longitudinal axis (ex. femur_1 was scaled using LLKnee and LTroch).

- Bony landmarks were set to a weight of 5, while clusters were set to a weight of 2

- Cluster position was adjusted to match experimental data. Other marker positions were adjusted as necessary. "Preview static pose" was selected until error values were satisfactory. 
- Process was continued until RMS error was less than $0.01 \mathrm{~m}$ and maximum marker error less than $0.02 \mathrm{~m}$. "Preview static pose" was unchecked and model markers were adjusted to match experimental.

- Mass of subject was used for control model and initial scaling. External mass of the hexbar was added to scaled model as additional body mass, and model was rescaled without marker position changing. Each subject had separate control, 20\%,40\%, and $60 \%$ models.

\section{OpenSim - Inverse Kinematics}

- The time points of the concentric phase for each trial were obtained from Visual3D, and $0.05 \mathrm{~s}$ were added to either end for IK process.

- The lumbar, subtalar, and MTP joints were locked.

- Based on marker error, select markers with high tracking error (ex. trochanter markers) were not included in IK, while still maintaining a minimum of 3 markers per segment. RMS and maximum marker error were minimized.

OpenSim - Inverse Dynamics

- IK output was used as input, with trajectories filtered at $13 \mathrm{~Hz}$.

- GRF applied as point force to foot at point of contact with force plate. GRF from both force plates was used.

- $\quad 0.05 \mathrm{~s}$ buffer was removed at toe-off.

OpenSim - Static Optimization

- IK output was used as input, and external load file from ID analysis was used. $0.05 \mathrm{~s}$ buffer at toe-off was removed. 
- Reserve actuators were appended to the model force set. Optimal force of reserve actuators was initially set to 10 and reduced to a minimum while still finding a solution.

- From one trial to the next within a given condition, the previous reserve file was used as a starting point, and optimal forces were adjusted up or down as required.

OpenSim - Muscle Analysis

- The 'controls' file that was output from SO for each trial was used as controls for the MuscleAnalysis tool.

- $\quad$ Motion was set to IK results file, coordinates filtered at $13 \mathrm{~Hz}$

- Solve for equilibrium was checked to 'on'

- $\quad$ Time range was set to match SO time range

- Actuators and external loads files were the same as used for SO

- MusclesAnalysis was added to list of active analyses, and compute moments was checked to 'on'

Data Processing - MATLAB

- Custom scripts were used to read in SO force files (.sto) and find peak force of each muscle for each trial. Script requests user input of body mass of subject.

- Peak force was averaged across trials at each load in both absolute terms and relative to body weight. Average absolute and relative peak force values for each subject were output as Excel documents.

- A custom script was used to calculate muscle torques and contributions to the NJM. Script requests user input of body mass of the subject. 
For each trial, script reads in SO output, and then reads in each MA output file. Muscle torque is calculated as the product of force and moment arm.

- Each trial was normalized to 101 points, allowing for the average moment arm and muscle torque to be calculated for each condition.

- Contribution (for individual subject) was calculated by dividing the timeseries average muscle torque for a given condition by the average timeseries NJM for that condition, then multiplying by 100 .

- Moment arms, muscle torques, and contributions were written to Excel documents.

- Custom scripts were used to normalize angles (IK output), moments (ID output), and muscle forces to 101 points. Timeseries were averaged across trials for each loading condition. 